\title{
Dispersion-enhanced solute transport in a cell-seeded hollow fibre membrane bioreactor
}

\author{
Natalie C. Pearson \\ Mathematical Institute, University of Oxford, Andrew Wiles Building, Radcliffe Observatory Quarter, \\ Woodstock Road, Oxford OX2 6GG, UK \\ Rebecca J. Shipley \\ Biomechanical Engineering Group, Department of Mechanical Engineering, University College London, \\ Torrington Place, London WC1E 7JE, UK
}

Sarah L. Waters

Mathematical Institute, University of Oxford, Andrew Wiles Building, Radcliffe Observatory Quarter, Woodstock Road, Oxford OX2 6GG, UK

James M. Oliver (oliver@maths.ox.ac.uk)

Mathematical Institute, University of Oxford, Andrew Wiles Building, Radcliffe Observatory Quarter, Woodstock Road, Oxford OX2 6GG, UK

\begin{abstract}
We present a matched asymptotic analysis of the fluid flow and solute transport in a small aspect ratio hollow fibre membrane bioreactor. A two-dimensional domain is assumed for simplicity, enabling greater understanding of the typical behaviours of the system in a setup which is analytically tractable. The model permits analysis related to Taylor dispersion problems, and allows us to predict the dependence of the mean solute uptake and solute exposure time on key parameters such as the inlet fluid fluxes, porous membrane porosity and cell layer porosity and width, which could be controlled or measured experimentally.
\end{abstract}

Keywords: tissue engineering, asymptotic reduction, Taylor dispersion

\section{Introduction}

Tissue engineering has great potential to provide clinical replacements for damaged or diseased tissue, as an alternative to existing therapies such as artificial implants and donor organs. It could help to overcome the significant current limitations such as the chronic shortage of donor tissue, and limited lifespan and lack of biocompatibility of synthetic joints [1]. Additional advantages over present approaches include reduced risk of infection or rejection by the patient's body [2]. However, progress to date has been hindered by the immense cost associated with the technique, preventing scale up for clinical use. Furthermore, the sensitivity of cells to their surrounding environment, and the vast range of cell types under consideration, mean that no one method will work for every application. Instead, optimal bioreactor design and operating conditions must be determined separately for each tissue type $[3,4]$.

One of the key elements of any dynamic bioreactor setup is the flow of culture media through the system, ensuring, for instance, sufficient nutrient delivery to the seeded cell population. Although the solute concentrations entering and leaving the bioreactor can be carefully controlled and measured, determining the exact solute distribution within the system is not possible experimentally. Mathematically modelling the flow and concentration profiles can therefore be extremely beneficial in determining experimental conditions under which cells will be most likely to thrive. Such models can provide detailed spatial and temporal information, for example highlighting regions of cells which are more likely to be nutrient-deprived.

The system considered in this paper is a hollow fibre membrane bioreactor (HFMB) shown in Figure 1. This consists of a cylindrical glass module with an open port at each end of the extracapillary space (ECS). A porous hollow fibre is inserted through the centre and fixed at both ends, and cells are seeded within the ECS between the fibre and module wall; various seeding configurations are used experimentally. In the flow regime which we consider here, fluid

(c) 2014 Kluwer Academic Publishers. Printed in the Netherlands. 


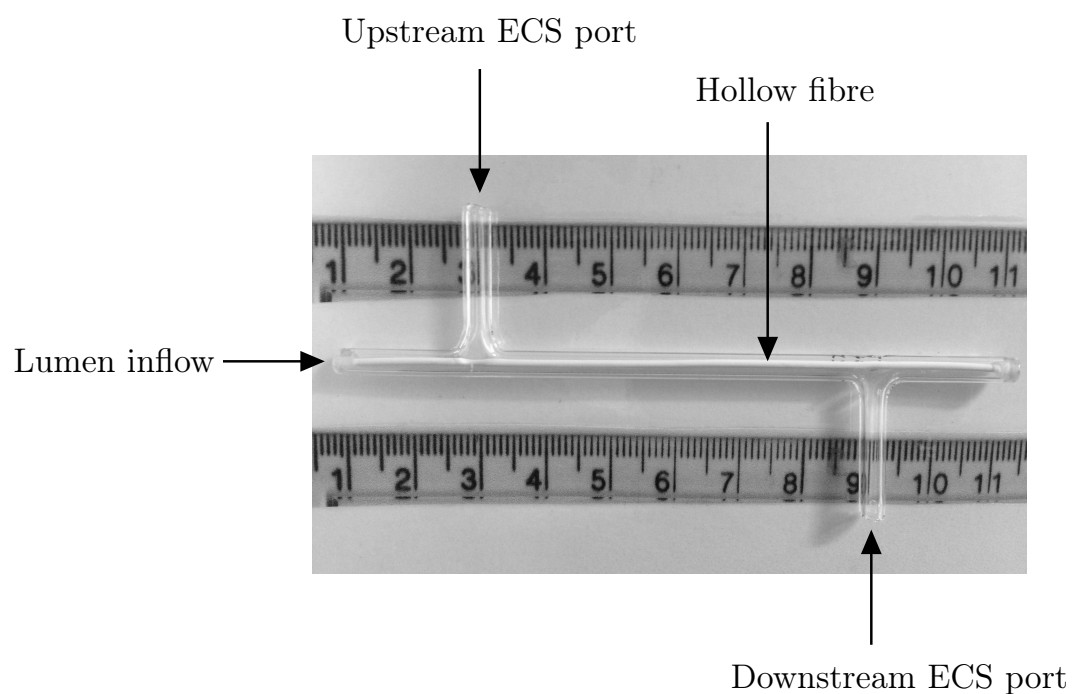

Figure 1. Photograph of a single HFMB module (ruler scale in $\mathrm{cm}$ ). The ECS ports can be seen, along with the hollow fibre which runs through the centre of the module. Cells are seeded in a natural scaffold on the outside of the fibre which partially fills the ECS.

is pumped in via both the lumen inlet and the upstream ECS port. It then either continues to flow down the lumen/ECS respectively, or permeates through the porous membrane, before eventually leaving through the lumen outlet or downstream ECS port. This system was modelled in [5], when the entire ECS was assumed to be filled with a scaffold seeded with cells. In this work we are concerned with an alternative experimental setup in which the cell-scaffold construct only fills part of the ECS, so that there is an additional exterior layer of free-flowing fluid around the construct. This provides further control over the mechanical and chemical stimulation of the cell population. It is unknown, however, whether there is sufficient delivery of nutrient to the cell population for a given experimental scenario, a question which we seek to address via mathematical modelling [6].

In [5], the flow rates used were sufficiently slow that it was appropriate to include cell proliferation, motility and death in the mathematical model. Here we consider flow rates such that the timescale of fluid transport in the lumen is on the order of minutes, and therefore much shorter than those of any cell kinetics (which are typically days). It is therefore appropriate to neglect these effects and model the cell-scaffold construct as a rigid, porous medium similarly to the fibre membrane. The only role of the cells in this scenario is to contribute to the solid fraction of the porous medium, and to take up a solute which is supplied to the system at the upstream ends of the lumen and exterior fluid. Previous models which have taken this approach include work by Shipley and co-authors [7,8]. In [7], transport of oxygen through a HFMB was modelled in an axisymmetric geometry. Taking into account the small aspect ratio of the lumen, the relevant reduced Péclet number was assumed to be of order unity, so that mass transport occurred by both advection and diffusion in the lumen. The ECS ports were closed, and diffusion assumed to be the sole transport mechanism in the porous membrane and cellfilled ECS. Uptake of oxygen was modelled using Michaelis-Menten kinetics, and the system investigated using analytical methods in the high oxygen concentration limit, and numerical methods otherwise. This analysis provided a strategy to determine optimal values for the lumen velocity, lumen length or ECS depth which ensured sufficient oxygen throughout the system, for cell types with either a high or low oxygen requirement. An axisymmetric configuration was also considered in [8], who again assumed the relevant reduced Péclet number to be of order unity. 
However, in this work flow through both the porous membrane and cell-packed ECS was included and modelled using Darcy's law. Concentrations of both oxygen and lactate were tracked, with a constant rate of uptake and production respectively. Two experimental configurations were considered, with the ECS ports either open or closed, and operating conditions were found in each case to ensure required bounds on the solute concentrations were satisfied.

In this paper we build on the work of [5] by considering the as yet unexplored, and physically relevant, regime in which the reduced Péclet number is comparable in size to the small lumen aspect ratio. In this limit it is still appropriate to consider the cell population as fixed as discussed above, and the effect of advection on the solute concentration is more apparent than in our previous work (in which the relevant reduced Péclet number was much smaller). In fact it will be shown that the dispersion of solute is enhanced by the increased flow, in a manner analogous to classical Taylor dispersion [9-11]. This adds to the findings of Griffiths et al. [12], who determined a generalised dispersion coefficient for flow in a long, thin porous-walled tube. We note that other models have also considered the effect on Taylor dispersion of porous media, or porous boundaries (see, for example, [13-15]), and solute absorption [16-18], but are rarely motivated by questions relevant to tissue engineering scenarios.

\subsection{PAPER OUtLine}

The HFMB setup as described above involves a non-trivial, three-dimensional geometry with multiple compartments. In the central region, away from the ECS ports, we would expect a fully developed, axisymmetric flow assuming a uniformly seeded cell layer. However, analysis of the system in this central region would yield highly complicated algebra even in an axisymmetric geometry, inhibiting clear exposition of the underlying asymptotics. We instead consider a twodimensional Cartesian domain, given that the asymptotic analysis in this setup is analogous to that in the axisymmetric case. This permits a clearer presentation of results, demonstrating typical behaviours of the system. In practice the same analysis could be carried over to the full three-dimensional case.

We begin in $\S 2$ by describing the model setup in a two-dimensional geometry, along with governing equations and boundary conditions. In $\S 3$ we show that to make analytical progress a simplified geometry may be considered, which allows the dynamics of the bulk of the bioreactor region to be studied through solution of an outer problem. We discuss relevant parameter values in $\S 3.3$, which motivate our choice of non-dimensionalisation in $\S 3.4$. In $\S \S 3.5,3.6$ we solve for the flow velocities and solute concentrations in each section at leading- and first-order in the lumen aspect ratio. This includes specifying the axial boundary conditions which must be applied in order to match with the inner problems in the regions near the bioreactor inlet and outlet, details of which are given in Appendix A. In $\S 3.7$, in the special case of constant inlet concentration, we are able to determine explicit expressions for the solute concentration at leading- and first-order which can be used to verify subsequent numerical solutions. In $\S 3.8$ we sum the depth-averaged version of the leading- and first-order equations governing the solute concentration to obtain a single reaction-advection-diffusion equation suitable for efficient numerical simulations. This equation also allows us to put our matched asymptotic analysis in the context of Taylor dispersion, and in the limit of an impermeable membrane and cell layer the classical Taylor dispersion equations for flow in a two-dimensional pipe can be recovered. Results for the constant inlet concentration system are presented in $\S 4.1$, investigating the dependence of the first-order concentration in each section on key dimensionless parameters that are controllable experimentally. In $\S 4.2 .1$, we investigate the dependence of the effective diffusion coefficient on these key parameters. Finally, in $\S 4.2 .2$ we consider the effect these parameters have on the mean solute uptake and solute exposure time throughout the bioreactor, before concluding our findings in $\S 5$. 


\section{Model setup}

We consider the two-dimensional simplified HFMB shown schematically in Figure 2, in which $(x, y)$ are Cartesian coordinates. The sections $\Omega_{i}(i=1,2,3,4)$ respectively denote the lumen, porous membrane, cell layer and upper fluid layer, and $\partial \Omega_{i j}(i, j=1,2,3,4, i \neq j)$ denote the (permeable) interfaces between sections. The remaining (impermeable) section boundaries are denoted by $\partial \Omega_{i}(i=2,3,4)$. Finally $A, B, C$ and $D$ represent line segments across the inlet/outlet pipes a finite distance away from the lumen inlet, upstream ECS port, lumen outlet and downstream ECS port respectively. Upon moving into two dimensions, in order to mimic the full three-dimensional setup in which the ECS ports are connected to the entire ECS (as depicted in Figure 1), we have included two up- and two down-stream ECS ports in our twodimensional geometry. In anticipation of the asymptotic analysis which is to follow, we have labelled the central 'Outer' region in which we will solve for the outer solution. This will need to be matched with the inner solutions in the up- and down-stream 'Inner' regions of $\mathrm{O}(1)$ aspect ratio which include the complicated geometry of the ECS ports. For the purposes of this analysis we take the width of these inner regions to be comparable to their height, both of which are much smaller than the bioreactor length.

\subsection{Governing EQuAtions}

In the lumen and upper fluid layer $\left(\Omega_{1}\right.$ and $\left.\Omega_{4}\right)$, the reduced Reynolds number is much smaller than the lumen aspect ratio (this is confirmed a posteriori, see §3.3). For the work presented here, this means that inertial effects will only appear at higher orders than we consider (specifically, at second order in the lumen aspect ratio in $\S 3$, and first order in the lumen aspect ratio in Appendix A.1). We thus neglect these effects from the start for simplicity, so that the appropriate governing equations for the fluid flow in these sections are:

$$
\nabla \cdot \mathbf{u}_{i}=0, \quad \nabla \cdot \boldsymbol{\sigma}_{i}=0, \quad \boldsymbol{\sigma}_{i}=-p_{i} \mathbf{I}+\mu_{\mathrm{w}}\left(\nabla \mathbf{u}_{i}+\left(\nabla \mathbf{u}_{i}\right)^{\mathrm{T}}\right), \quad i=1, \mathrm{f},
$$

where $\mathbf{u}_{i}=\left(u_{i}, v_{i}\right)$ and $\boldsymbol{\sigma}_{i}(i=1, \mathrm{f})$ respectively denote the fluid velocity and fluid stress tensor in the lumen/upper fluid layer. In addition, $p_{i}(i=1, \mathrm{f})$ represents the reduced fluid pressure in each section, defined in terms of the absolute fluid pressure $\bar{p}_{i}$ by $p_{i}=\bar{p}_{i}+\rho_{\mathrm{w}} g y(i=1, \mathrm{f})$ as the effect of gravity cannot be neglected at the low flow rates we will consider (see §3.3). The fluid viscosity $\mu_{\mathrm{w}}$ is assumed to be constant, and equal to that of water.

The porous membrane $\Omega_{2}$ is assumed to have constant permeability $k_{\mathrm{m}}$ and porosity $\phi_{\mathrm{m}}$. As mentioned above, we consider the cell layer to be a porous medium consisting of the cells and a scaffold; on the timescale of interest for our analysis, the cells do not move, proliferate or die, and therefore purely contribute volume to the porous domain. Therefore we also assume constant permeability $k_{\mathrm{w}}$ and porosity $\phi_{\mathrm{w}}$ of the cell layer $\left(\Omega_{3}\right)$. Hence in the membrane and cell layer, the governing equations are those for Darcy flow in a porous medium:

$$
\nabla \cdot \mathbf{u}_{i}=0, \quad \mathbf{u}_{i}=-\frac{k_{i}}{\mu_{\mathrm{w}}} \nabla p_{i}, \quad i=\mathrm{m}, \mathrm{w},
$$

where $\mathbf{u}_{i}=\left(u_{i}, v_{i}\right)$ and $p_{i}(i=\mathrm{m}, \mathrm{w})$ are respectively the fluid velocity and reduced fluid pressure in the membrane/cell layer (with the reduced pressures defined as above).

Mass transport is described by the conservation of mass advection-diffusion equation in each section. Additionally, in the cell layer we use a reaction term $\mathcal{R}$ to represent solute uptake per unit volume of mixture by the cells, and assume that solute transport only occurs in the water. We will assume constant uptake provided the solute concentration is non-zero, i.e. we set $\mathcal{R}=0$ if $c_{\mathrm{w}}=0$. We note that this constant uptake can be derived from the high concentration limit of Michaelis-Menten kinetics; although it is not clear if the concentration is always sufficiently 


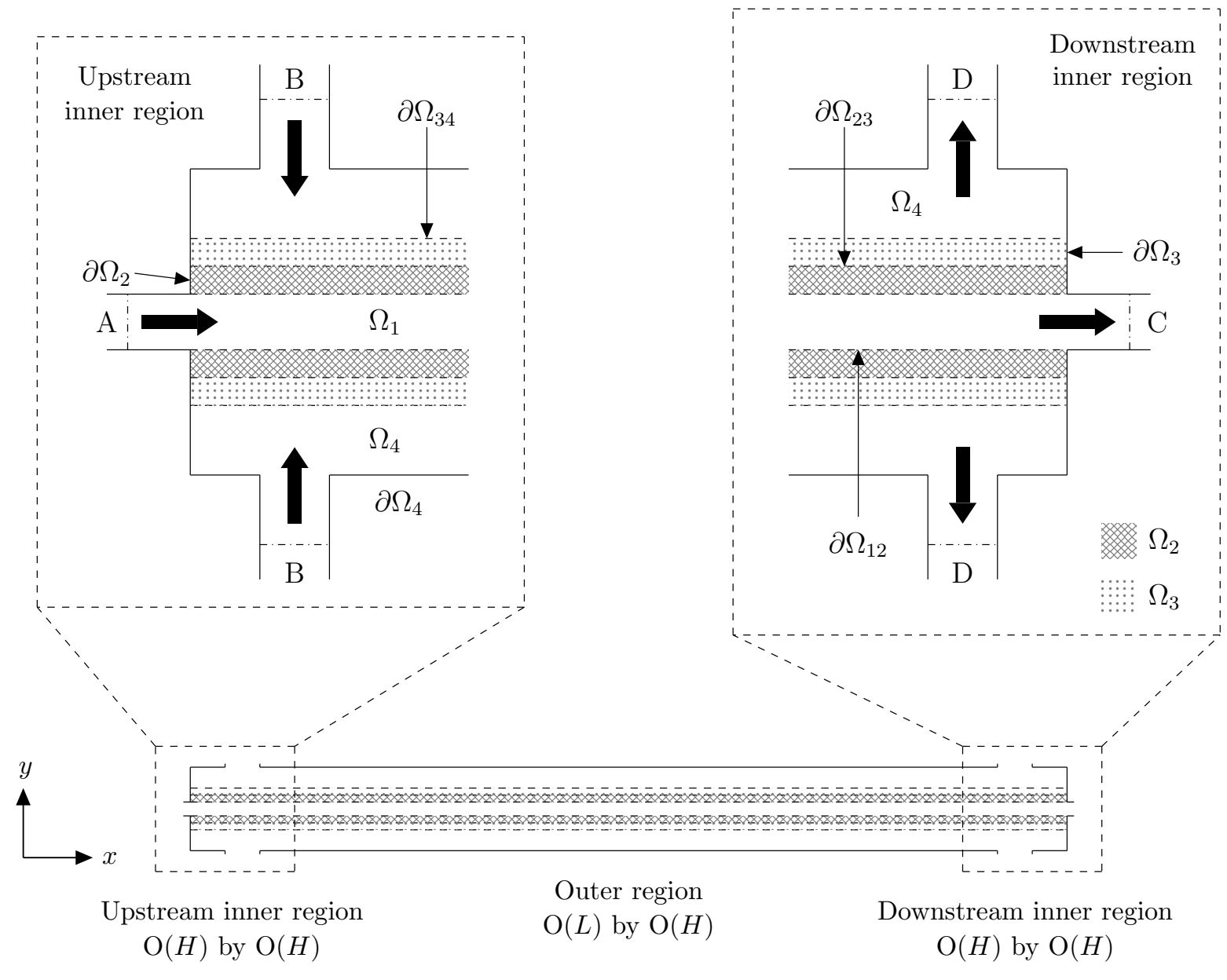

Figure 2. Simplified modelling domain in a two-dimensional Cartesian geometry, with the up- and down-stream inner regions near the ECS ports enlarged (not to scale). The solid black arrows denote the direction of fluid flow. The lumen, porous membrane, cell layer and upper fluid layer respectively occupy $\Omega_{i}(i=1,2,3,4)$. Dashed lines represent permeable boundaries $\partial \Omega_{i j}$ between sections $\Omega_{i}$ and $\Omega_{j}$, and solid lines denote impermeable section boundaries $\partial \Omega_{i}$ where applicable. The dash-dotted lines $A, B, C$ and $D$ respectively denote line segments across the width of the pipes a finite distance away from the lumen inlet, upstream ECS ports, lumen outlet and downstream ECS ports respectively. Here $H$ and $L, H \ll L$, are typical vertical and horizontal length scales respectively, and will be specified during the asymptotic analysis in $\S 3$.

high here, we retain this form for simplicity. Thus we have

$$
\begin{aligned}
\frac{\partial c_{\mathrm{i}}}{\partial t}+\nabla \cdot\left(c_{i} \mathbf{u}_{i}\right)= & D \nabla^{2} c_{i}, \quad i=1, \mathrm{f}, \quad \frac{\partial\left(\phi_{\mathrm{m}} c_{\mathrm{m}}\right)}{\partial t}+\nabla \cdot\left(\phi_{\mathrm{m}} c_{\mathrm{m}} \mathbf{u}_{\mathrm{m}}\right)=\phi_{\mathrm{m}} D \nabla^{2} c_{\mathrm{m}}, \\
& \frac{\partial\left(\phi_{\mathrm{w}} c_{\mathrm{w}}\right)}{\partial t}+\nabla \cdot\left(\phi_{\mathrm{w}} c_{\mathrm{w}} \mathbf{u}_{\mathrm{w}}\right)=\phi_{\mathrm{w}} D \nabla^{2} c_{\mathrm{w}}-\mathcal{R},
\end{aligned}
$$

where $c_{i}$ (for $i=1, \mathrm{~m}, \mathrm{w}, \mathrm{f}$ ) is the concentration per unit volume of fluid in each section and $D$ is the (constant) diffusion coefficient of the solute in water, assumed to be of equal value in each section. 


\subsection{BOUNDARY CONDITIONS}

We now impose boundary conditions to couple the sections and close the problem. On each of the interfaces between sections, we impose continuity of fluid flux, of normal stress, of solute concentration and of solute flux. In addition, the lumen/membrane and cell/upper fluid layer interfaces are at the boundary between free fluid flow and porous media flow and hence need an extra condition; in both cases this is given by no slip of fluid in the free flowing section.

In the following, $\mathbf{t}_{i j}$ is the unit tangent to $\partial \Omega_{i j}, \mathbf{n}_{i}$ is the outward unit normal to $\partial \Omega_{i}$ and $\mathbf{n}_{i j}$ is the unit normal pointing into section $\Omega_{j}$. We begin with the lumen/membrane interface $\partial \Omega_{12}$, where we impose no slip of fluid, continuity of fluid flux and of normal stress, and continuity of concentration and of solute flux:

$$
\begin{gathered}
\mathbf{u}_{1} \cdot \mathbf{t}_{12}=0, \quad \mathbf{u}_{1} \cdot \mathbf{n}_{12}=\phi_{\mathrm{m}} \mathbf{u}_{\mathrm{m}} \cdot \mathbf{n}_{12}, \quad \mathbf{n}_{12} \cdot \boldsymbol{\sigma}_{\mathrm{l}} \cdot \mathbf{n}_{12}=\mathbf{n}_{12} \cdot \boldsymbol{\sigma}_{\mathrm{m}} \cdot \mathbf{n}_{12}, \\
c_{\mathrm{l}}=c_{\mathrm{m}}, \quad \nabla c_{\mathrm{l}} \cdot \mathbf{n}_{12}=\phi_{\mathrm{m}} \nabla c_{\mathrm{m}} \cdot \mathbf{n}_{12} \quad \text { on } \Omega_{12},
\end{gathered}
$$

where $\boldsymbol{\sigma}_{\mathrm{m}}=-p_{\mathrm{m}} \mathbf{I}$ and we have assumed that all the stress is taken up by the water in the membrane. The no slip condition is motivated by the results from [19], in which the authors found that the effect of slip is insignificant for the porous membranes used experimentally in tissue engineering scenarios. On the membrane/cell layer and cell/upper fluid interfaces $\partial \Omega_{23}$, $\partial \Omega_{34}$, we impose continuity of fluid flux, of normal stress, of concentration and of solute flux:

$$
\begin{gathered}
\phi_{\mathrm{m}} \mathbf{u}_{\mathrm{m}} \cdot \mathbf{n}_{23}=\phi_{\mathrm{w}} \mathbf{u}_{\mathrm{w}} \cdot \mathbf{n}_{23}, \quad \mathbf{n}_{23} \cdot \boldsymbol{\sigma}_{\mathrm{m}} \cdot \mathbf{n}_{23}=\mathbf{n}_{23} \cdot \boldsymbol{\sigma}_{\mathrm{w}} \cdot \mathbf{n}_{23}, \\
c_{\mathrm{m}}=c_{\mathrm{w}}, \quad \phi_{\mathrm{m}} \nabla c_{\mathrm{m}} \cdot \mathbf{n}_{23}=\phi_{\mathrm{w}} \nabla c_{\mathrm{w}} \cdot \mathbf{n}_{23} \quad \text { on } \partial \Omega_{23}, \\
\phi_{\mathrm{w}} \mathbf{u}_{\mathrm{w}} \cdot \mathbf{n}_{34}=\mathbf{u}_{\mathrm{f}} \cdot \mathbf{n}_{34}, \quad \mathbf{n}_{34} \cdot \boldsymbol{\sigma}_{\mathrm{w}} \cdot \mathbf{n}_{34}=\mathbf{n}_{34} \cdot \boldsymbol{\sigma}_{\mathrm{f}} \cdot \mathbf{n}_{34}, \\
c_{\mathrm{w}}=c_{\mathrm{f}}, \quad \phi_{\mathrm{w}} \nabla c_{\mathrm{w}} \cdot \mathbf{n}_{34}=\nabla c_{\mathrm{f}} \cdot \mathbf{n}_{34} \quad \text { on } \partial \Omega_{34},
\end{gathered}
$$

where $\boldsymbol{\sigma}_{\mathrm{w}}=-p_{\mathrm{w}} \mathbf{I}$, and again we have assumed that the water takes up all the stress. In addition, we also prescribe no slip of fluid on the cell/upper fluid interface:

$$
\mathbf{u}_{\mathrm{f}} \cdot \mathbf{t}_{34}=0 \quad \text { on } \partial \Omega_{34} .
$$

On the edges of the bioreactor $\partial \Omega_{i}(i=2,3,4)$ we impose no flux of fluid and, on $\partial \Omega_{4}$, no slip of fluid and no flux of solute:

$$
\begin{aligned}
& \mathbf{u}_{\mathrm{m}} \cdot \mathbf{n}_{2}=0, \quad \nabla c_{\mathrm{m}} \cdot \mathbf{n}_{2}=0 \quad \text { on } \partial \Omega_{2}, \\
& \mathbf{u}_{\mathrm{w}} \cdot \mathbf{n}_{3}=0, \quad \nabla c_{\mathrm{w}} \cdot \mathbf{n}_{3}=0 \quad \text { on } \partial \Omega_{3}, \\
& \mathbf{u}_{\mathrm{f}}=0, \quad \nabla c_{\mathrm{f}} \cdot \mathbf{n}_{4}=0 \quad \text { on } \partial \Omega_{4} .
\end{aligned}
$$

It now remains to prescribe boundary conditions at the lumen inlet and outlet, and the ECS ports. In the pipes entering the lumen inlet and upstream ECS port, we assume that the flow is of Poiseuille form with prescribed volume fluxes in per unit length in the $z$-direction (perpendicular to both $x$ and $y) Q_{1, \text { in }}$ and $Q_{\mathrm{f} \text {,in }}$ respectively. We also prescribe the solute concentration at these points, so that

$$
\begin{gathered}
c_{\mathrm{l}}=c_{\mathrm{l}, \text { in }}(t) \quad \text { at } A, \\
c_{\mathrm{f}}=c_{\mathrm{f}, \text { in }}(t)
\end{gathered}
$$

for some $c_{\mathrm{l} \text {,in }}$ and $c_{\mathrm{f} \text {,in }}$ which (for now) are assumed to be functions of time. Experimentally, the lumen outlet pressure is controlled by a clamp, and the pipe connected to the downstream ECS port is left open to the atmosphere. We prescribe continuity of normal stress and an axial flow at these positions, and thus the appropriate conditions are

$$
\begin{array}{lrl}
\mathbf{n}_{\mathrm{C}} \cdot \boldsymbol{\sigma}_{\mathrm{l}} \cdot \mathbf{n}_{\mathrm{C}}=P_{\mathrm{d}}, & \mathbf{u}_{1} \cdot \mathbf{t}_{\mathrm{C}}=0 & \text { at } C, \\
\mathbf{n}_{\mathrm{D}} \cdot \boldsymbol{\sigma}_{\mathrm{f}} \cdot \mathbf{n}_{\mathrm{D}}=p_{\text {atm }}, & \mathbf{u}_{\mathrm{f}} \cdot \mathbf{t}_{\mathrm{D}}=0 & \text { at } D,
\end{array}
$$


where $P_{\mathrm{d}}$ is a given constant downstream pressure, $p_{\text {atm }}$ is atmospheric pressure, $\mathbf{n}_{\mathrm{C}} / \mathbf{t}_{\mathrm{C}}, \mathbf{n}_{\mathrm{D}} / \mathbf{t}_{\mathrm{D}}$ are the outward pointing normals/tangents at $C$ and $D$ respectively, and $\boldsymbol{\sigma}_{i}(i=1, \mathrm{f})$ respectively are the fluid stress tensors in the lumen and upper fluid layer. Finally, we must impose a condition on the solute concentration at positions $C$ and $D$. There is no constraint here experimentally, and so we prescribe no diffusive flux of solute. This is the condition that will have the least effect on the theoretical results, and is motivated by the expectation that the solute concentration is constant in space as it leaves the bioreactor due to the effect of diffusion; this is borne out by our subsequent boundary layer analysis in Appendix B. Hence we have

$$
\begin{aligned}
\nabla c_{\mathrm{l}} \cdot \mathbf{n}_{\text {out }}=0 & \text { at } C, \\
\nabla c_{\mathrm{f}} \cdot \mathbf{n}_{\mathrm{d}}=0 & \text { at } D,
\end{aligned}
$$

where $\mathbf{n}_{\text {out }}$ and $\mathbf{n}_{\mathrm{d}}$ respectively are the unit normals in the direction of fluid flow at the lumen outlet and downstream ECS port.

\section{Outer solution}

The geometry of the modelling domain as illustrated in Figure 2 is complicated by the ECS ports both up- and down-stream. Numerical approaches would be needed in order to make any progress in solving this system, and this would be computationally expensive. As mentioned in $\S 2$ above, we will instead concentrate on determining the solution in the outer region, excluding the ECS ports, as it greatly simplifies the geometry yet still incorporates the majority of the bioreactor. This will be sufficient to obtain a general understanding of the fluid flow and solute distribution in this system, and their dependence on certain key experimental parameters. The up- and down-stream boundary conditions to be applied on this outer problem are determined by matching with the inner solutions in the regions near the bioreactor inlet and outlet, details of which are given in Appendix A.

\subsection{Geometry For the outer solution}

The modelling domain for the outer solution is depicted in Figure 3. Given the symmetry of the two-dimensional system depicted in Figure 2, we consider the upper half of the bioreactor only. The coordinate system is fixed from the full setup in Figure 2: the origin $(x, y)=(0,0)$ is taken to be at the far left of the full domain, on the lumen centreline. Thus (in the limit $\varepsilon \rightarrow 0$, where $\varepsilon$ is the small lumen aspect ratio) the bottom left-hand corner of the outer region corresponds to $(x, y)=\left(0^{+}, 0\right)$, with the axial dimension of the bioreactor defined by $0^{+}<x<L^{-}$. The lumen then occupies $0<y<h_{1}$ in the transverse direction and the membrane $h_{1}<y<h_{1}+h_{2}$. We take the cell layer to be of comparable thickness to the membrane, defined by $h_{1}+h_{2}<y<h_{1}+h_{2}+h_{3}=H-h_{4}$, and the upper fluid layer occupies the remaining section $H-h_{4}<y<H$.

\subsection{GOVERning EQUATIONS AND BOUNDARY CONDITIONS FOR THE OUTER SOLUTION}

The governing equations in each section are unchanged from $\S 2.1$, as are the boundary conditions on the interfaces between sections and on the outer bioreactor boundary $\partial \Omega_{4}$. Only the boundary conditions at the up- and down-stream ends of this central outer region are altered. These can be determined from matching with the corresponding inner solutions at each end, details of which are given in Appendix A. In the following the resulting matching conditions will be introduced as required. In addition, as we now consider the upper-half of the outer region only, we require the following symmetry conditions on the lumen centreline $y=0$ :

$$
\frac{\partial u_{1}}{\partial y}=0, \quad v_{1}=0, \quad \frac{\partial c_{1}}{\partial y}=0 \quad \text { on } \quad y=0 .
$$




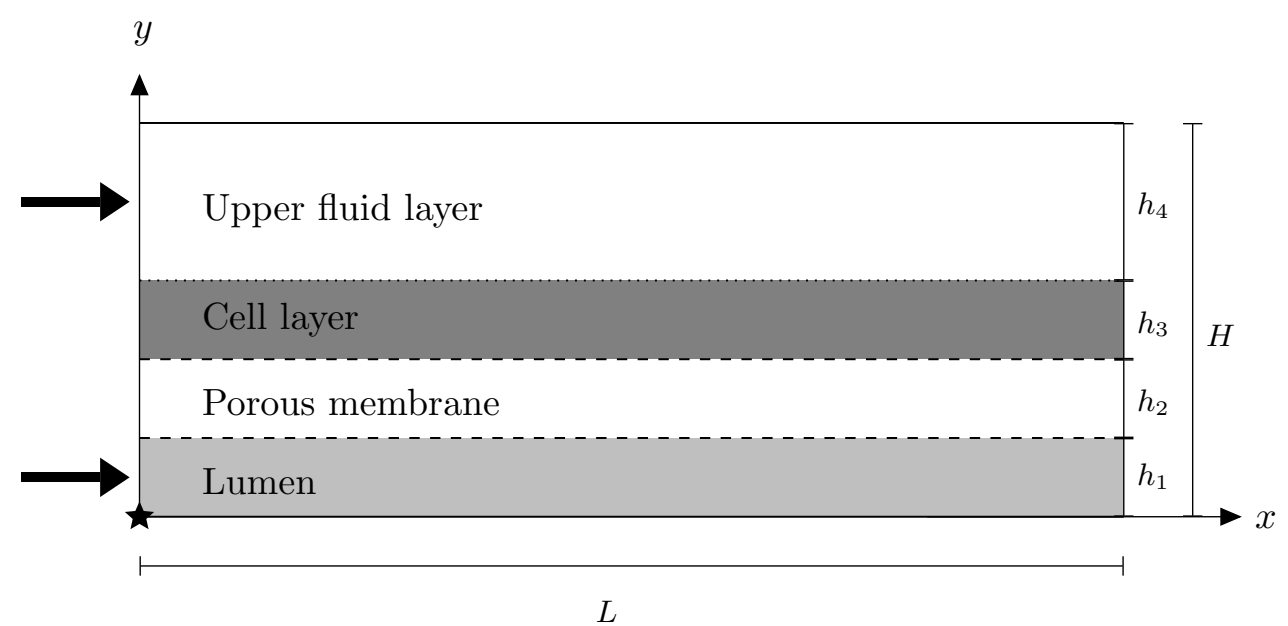

Figure 3. Upper-central bioreactor region representing the modelling domain for the outer problem in a two-dimensional Cartesian geometry (not to scale). The star denotes the point $(x, y)=\left(0^{+}, 0\right)$ and solid black arrows denote the direction and location of the fluid flow into this region.

\subsection{PARAmeter VAlues}

Dimensional and dimensionless parameter values for the model are given in Tables I and II, respectively. Where possible, values have been taken from the literature, or estimated based on discussions with experimentalists [6]. The lumen aspect ratio is given by $\varepsilon=h_{1} / L \ll 1$. The two-dimensional fluxes into the lumen and upper fluid layer have been determined from the corresponding three-dimensional values by first calculating the corresponding velocity and then multiplying by the length scale in the $y$-direction, $\varepsilon L$. These three-dimensional values have also been used to calculate the porous membrane/cell layer velocity scaling $U^{*}$. The fluxes have been chosen within an experimentally relevant range (which can vary greatly [6]) and such that the asymptotic bounds on the reduced Reynolds number $\varepsilon^{2} \mathrm{Re}=\varepsilon \rho_{\mathrm{w}} L U^{*} / \mu_{\mathrm{w}}$ and reduced Péclet number $\varepsilon^{2} \mathrm{Pe}=\varepsilon L U^{*} / D$ for this distinguished limit are satisfied. The concentration scaling $C^{*}$ is taken to be a typical inlet concentration for oxygen used in culturing a variety of cell types [8]. The dimensionless inlet concentrations $c_{1, \text { in }}$ and $c_{\mathrm{f}, \text { in }}$ are thus assumed to be of $\mathrm{O}(1)$. Finally, the reaction term $\mathcal{R}$ can also vary in size significantly; here a representative value is taken which corresponds to the rate of oxygen uptake in bovine chondrocytes [8].

In some cases either we have not been able to obtain experimental values, or a wide range of possible values exists. In such situations we make choices which retain the greatest number of features at leading order in our model. Specifically, the values of the cell layer permeability $k_{\mathrm{w}}$ and porosity $\phi_{\mathrm{w}}$ will vary greatly depending on the type of scaffold and cell used, and the seeding regime employed. We have therefore taken $k_{\mathrm{w}}$ such that its dimensionless equivalent $\kappa_{\mathrm{W}}$ is of $\mathrm{O}(1)$ in order to keep the model as general as possible. The porosity $\phi_{\mathrm{w}}$ must take values between 0 and 1 , and in what follows we will examine its influence on the results of the model. When its value must be fixed, we take $\phi_{\mathrm{w}}=0.54$ based on calculations by Shipley and Waters in $[8]$.

\subsection{NOn-Dimensionalisation}

We use the same scalings in the upper fluid layer as in the lumen, since both are supplied with a prescribed flux of fluid at the upstream end. Given that inertial effects are negligible compared with viscosity (see $\S 3.3$ ), we employ a viscous pressure scaling throughout. We assume a horizontal velocity scale of $U^{*}$ in the membrane and cell layer, and of $U^{*} / \varepsilon$ in the lumen and upper fluid layer, where $\varepsilon=h_{1} / L \ll 1$ is the lumen aspect ratio. As well as allowing the same 
Table I. Dimensional parameters.

\begin{tabular}{ll}
\hline Parameter & Dimensional value and units \\
\hline$h_{1}$ & $200 \mu \mathrm{m}^{\mathrm{a}}$ \\
$h_{2}$ & $200 \mu \mathrm{m}^{\mathrm{a}}$ \\
$h_{3}+h_{4}$ & $600 \mu \mathrm{m}^{\mathrm{a}}$ \\
$L$ & $0.1 \mathrm{~m}^{\mathrm{a}}$ \\
$\rho_{\mathrm{w}}$ & $1 \mathrm{~g} \mathrm{~cm}^{-3 \mathrm{a}}$ \\
$\mu_{\mathrm{w}}$ & $10^{-3} \mathrm{~Pa} \mathrm{~s}^{\mathrm{a}}$ \\
$k_{\mathrm{m}}$ & $6.73 \times 10^{-16} \mathrm{~m}^{2} \mathrm{~b}$ \\
$p_{\mathrm{atm}}$ & $14.69 \mathrm{psia}^{\mathrm{a}}$ \\
$Q_{1, \text { in }}, Q_{\mathrm{f}, \text { in }}$ & $5 \times 10^{-5}-5 \times 10^{-2} \mathrm{ml} \mathrm{min}^{-1 \mathrm{c}}$ \\
$D$ & $3 \times 10^{-9} \mathrm{~m}^{2} \mathrm{~s}^{-1 \mathrm{a}}$ \\
$\mathcal{R}$ & $4.8 \times 10^{-5} \mathrm{~mol} \mathrm{~m}^{-3} \mathrm{~s}^{-1 \mathrm{~d}}$ \\
$U^{*}$ & $1.33 \times 10^{-6} \mathrm{~m} \mathrm{~s}^{-1 ~ c}$ \\
$C^{*}$ & $0.22 \mathrm{~mol} \mathrm{~m}^{-3} \mathrm{a}$ \\
\end{tabular}

${ }^{a}$ values taken from [19]

${ }^{\mathrm{b}}$ experimentally obtained values

${ }^{\mathrm{c}}$ values based on estimations by our experimental collaborators

$\mathrm{d}$ values taken from [8]

Table II. Dimensionless parameters, along with any bounds imposed either physically or by the asymptotic analysis.

\begin{tabular}{llll}
\hline Parameter & Definition & Value & Restriction (if any) \\
\hline$\hat{h}_{2}$ & $h_{2} /(\varepsilon L)$ & 1 & $\hat{h}_{2}>0$ \\
$\hat{h}_{3}+\hat{h}_{4}$ & $\left(h_{3}+h_{4}\right) /(\varepsilon L)$ & 3 & $\hat{h}_{3}, \hat{h}_{4}>0$ \\
$\varepsilon$ & $h_{1} / L$ & $2 \times 10^{-3}$ & $0<\varepsilon \ll 1$ \\
$\varepsilon^{2} \mathrm{Pe}$ & $\varepsilon L U^{*} / D$ & 0.0884 & $\varepsilon^{2} \ll \varepsilon^{2} \mathrm{Pe} \ll 1$ \\
$\varepsilon^{2} \operatorname{Re}$ & $\varepsilon \rho_{\mathrm{w}} L U^{*} / \mu_{\mathrm{w}}$ & $2.66 \times 10^{-4}$ & $\varepsilon^{2} \operatorname{Re} \ll \varepsilon$ \\
$\phi_{\mathrm{m}}$ & - & $0.77^{\mathrm{a}}$ & $0<\phi_{\mathrm{m}}<1$ \\
$\phi_{\mathrm{w}}$ & - & $0.54^{\mathrm{b}}$ & $0<\phi_{\mathrm{w}}<1$ \\
$\kappa_{\mathrm{m}}$ & $k_{\mathrm{m}} /\left(\varepsilon^{5} L^{2}\right)$ & 2.1 & $\varepsilon \ll \kappa_{\mathrm{m}} \ll 1 / \varepsilon$ \\
$\kappa_{\mathrm{w}}$ & $k_{\mathrm{w}} /\left(\varepsilon^{5} L^{2}\right)$ & 1 & $\varepsilon \ll \kappa_{\mathrm{w}} \ll 1 / \varepsilon$ \\
$\hat{Q}_{i, \text { in }}, i=1, \mathrm{f}$ & $\lambda Q_{i, \text { in }} /\left(L U^{*}\right)$ & $0.01-10$ & $\varepsilon \ll \hat{Q}_{i, \text { in }} \ll 1 / \varepsilon$ \\
$\hat{\mathcal{R}}$ & $\varepsilon L^{2} \mathcal{R} /\left(D C^{*}\right)$ & 1.454 & $\varepsilon \ll \hat{\mathcal{R}}_{1} \ll 1 / \varepsilon$ \\
$\hat{c}_{1, \text { in }}$ & $c_{1, \text { in }} / C^{*}$ & 1 & $\varepsilon \ll \hat{c}_{1, \text { in }} \ll 1 / \varepsilon$ \\
$\hat{c}_{\mathrm{f}, \text { in }}$ & $c_{\mathrm{f}, \text { in }} / C^{*}$ & 1 & $\varepsilon \ll \hat{c}_{\mathrm{f}, \text { in }} \ll 1 / \varepsilon$ \\
\hline
\end{tabular}

values taken from ${ }^{\mathrm{a}}[21]$ and ${ }^{\mathrm{b}}[8]$

pressure scales to be applied in each section, this scaling is motivated by the fact that the flow in the membrane and cell layer will be much smaller than in the outer layers due to the resistance to flow within these porous sections. We set the timescale of interest to be that for advection in the lumen $(\approx 2.5$ mins given the choice of velocity scale in Table I). We also expect to see the effects of solute uptake on this timescale and scale the reaction term $\mathcal{R}$ accordingly; the exact value corresponds to the rate of oxygen uptake by bovine chondrocytes [8]. Hence the relevant 
non-dimensionalisation is as follows:

$$
\begin{gathered}
x=L \hat{x}, \quad y=\varepsilon L \hat{y}, \quad h_{i}=\varepsilon L \hat{h}_{i} \quad(i=2,3,4), \quad H=\varepsilon L \hat{H}, \quad t=\frac{\varepsilon L}{U^{*}} \hat{t}, \\
u_{i}=\frac{U^{*}}{\varepsilon} \hat{u}_{i}, \quad v_{i}=U^{*} \hat{v}_{i} \quad(i=1, \mathrm{f}), \quad u_{i}=U^{*} \hat{u}_{i}, \quad v_{i}=\varepsilon U^{*} \hat{v}_{i} \quad(i=\mathrm{m}, \mathrm{w}), \\
p_{i}=p_{\mathrm{atm}}+\frac{\mu_{\mathrm{w}} U^{*}}{\varepsilon^{3} L} \hat{p}_{i}, \quad c_{i}=C^{*} \hat{c}_{i} \quad(i=1, \mathrm{~m}, \mathrm{w}, \mathrm{f}), \quad \mathcal{R}=\frac{D C^{*}}{\varepsilon L^{2}} \hat{\mathcal{R}} .
\end{gathered}
$$

Dropping hats on dimensionless variables and substituting in for $p_{\mathrm{m}}$ and $p_{\mathrm{w}}$ in place of $u_{\mathrm{m}}, v_{\mathrm{m}}$, $u_{\mathrm{w}}$ and $v_{\mathrm{w}}$ where relevant, the governing equations are, in the lumen

$$
\begin{array}{cl}
\frac{\partial u_{1}}{\partial x}+\frac{\partial v_{1}}{\partial y}=0, & -\frac{\partial p_{1}}{\partial x}+\varepsilon^{2} \frac{\partial^{2} u_{1}}{\partial x^{2}}+\frac{\partial^{2} u_{1}}{\partial y^{2}}=0, \quad-\frac{\partial p_{1}}{\partial y}+\varepsilon^{4} \frac{\partial^{2} v_{1}}{\partial x^{2}}+\varepsilon^{2} \frac{\partial^{2} v_{1}}{\partial y^{2}}=0, \\
\varepsilon^{2} \operatorname{Pe}\left(\frac{\partial c_{1}}{\partial t}+\nabla \cdot\left(c_{1} \mathbf{u}_{1}\right)\right)=\varepsilon^{2} \frac{\partial^{2} c_{1}}{\partial x^{2}}+\frac{\partial^{2} c_{1}}{\partial y^{2}} ;
\end{array}
$$

in the porous membrane

$$
\begin{gathered}
u_{\mathrm{m}}=-\varepsilon^{2} \kappa_{\mathrm{m}} \frac{\partial p_{\mathrm{m}}}{\partial x}, \quad v_{\mathrm{m}}=-\kappa_{\mathrm{m}} \frac{\partial p_{\mathrm{m}}}{\partial y}, \quad \varepsilon^{2} \frac{\partial^{2} p_{\mathrm{m}}}{\partial x^{2}}+\frac{\partial^{2} p_{\mathrm{m}}}{\partial y^{2}}=0, \\
\varepsilon^{2} \operatorname{Pe}\left(\frac{\partial c_{\mathrm{m}}}{\partial t}+\varepsilon \nabla \cdot\left(c_{\mathrm{m}} \mathbf{u}_{\mathrm{m}}\right)\right)=\varepsilon^{2} \frac{\partial^{2} c_{\mathrm{m}}}{\partial x^{2}}+\frac{\partial^{2} c_{\mathrm{m}}}{\partial y^{2}}
\end{gathered}
$$

in the cell layer

$$
\begin{aligned}
u_{\mathrm{w}}= & -\varepsilon^{2} \kappa_{\mathrm{w}} \frac{\partial p_{\mathrm{w}}}{\partial x}, \quad v_{\mathrm{w}}=-\kappa_{\mathrm{w}} \frac{\partial p_{\mathrm{w}}}{\partial y}, \quad \varepsilon^{2} \frac{\partial^{2} p_{\mathrm{w}}}{\partial x^{2}}+\frac{\partial^{2} p_{\mathrm{w}}}{\partial y^{2}}=0, \\
& \varepsilon^{2} \operatorname{Pe}\left(\frac{\partial c_{\mathrm{w}}}{\partial t}+\varepsilon \nabla \cdot\left(c_{\mathrm{w}} \mathbf{u}_{\mathrm{w}}\right)\right)=\varepsilon^{2} \frac{\partial^{2} c_{\mathrm{w}}}{\partial x^{2}}+\frac{\partial^{2} c_{\mathrm{w}}}{\partial y^{2}}-\frac{\varepsilon \mathcal{R}}{\phi_{\mathrm{w}}}
\end{aligned}
$$

and in the upper fluid layer

$$
\begin{gathered}
\frac{\partial u_{\mathrm{f}}}{\partial x}+\frac{\partial v_{\mathrm{f}}}{\partial y}=0, \quad-\frac{\partial p_{\mathrm{f}}}{\partial x}+\varepsilon^{2} \frac{\partial^{2} u_{\mathrm{f}}}{\partial x^{2}}+\frac{\partial^{2} u_{\mathrm{f}}}{\partial y^{2}}=0, \quad-\frac{\partial p_{\mathrm{f}}}{\partial y}+\varepsilon^{4} \frac{\partial^{2} v_{\mathrm{f}}}{\partial x^{2}}+\varepsilon^{2} \frac{\partial^{2} v_{\mathrm{f}}}{\partial y^{2}}=0, \\
\varepsilon^{2} \operatorname{Pe}\left(\frac{\partial c_{\mathrm{f}}}{\partial t}+\nabla \cdot\left(c_{\mathrm{f}} \mathbf{u}_{\mathrm{f}}\right)\right)=\varepsilon^{2} \frac{\partial^{2} c_{\mathrm{f}}}{\partial x^{2}}+\frac{\partial^{2} c_{\mathrm{f}}}{\partial y^{2}} .
\end{gathered}
$$

Here $\kappa_{\mathrm{m}}=k_{\mathrm{m}} /\left(\varepsilon^{5} L^{2}\right)$ and $\kappa_{\mathrm{w}}=k_{\mathrm{w}} /\left(\varepsilon^{5} L^{2}\right)$ are the dimensionless permeabilities of the membrane and cell layer respectively, which we assume to be of $\mathrm{O}(1)$ so that the leading-order flow in both porous sections is in the transverse direction. The Péclet number for axial flow in the lumen and upper fluid layer is $\mathrm{Pe}=L U^{*} /(\varepsilon D)$, which we take to be of $\mathrm{O}(1 / \varepsilon)$ so that advection dominates axial diffusion in these sections. This is consistent with the range of flow rates used experimentally.

The dimensionless boundary conditions are as follows (again substituting in for $p_{\mathrm{m}}$ and $p_{\mathrm{w}}$ ):

$$
\begin{aligned}
\frac{\partial u_{\mathrm{l}}}{\partial y} & =0, \quad v_{\mathrm{l}}=0, \quad \frac{\partial c_{\mathrm{l}}}{\partial y}=0 \quad \text { on } y=0 ; \\
u_{\mathrm{l}}=0, \quad v_{\mathrm{l}} & =-\varepsilon \kappa_{\mathrm{m}} \phi_{\mathrm{m}} \frac{\partial p_{\mathrm{m}}}{\partial y}, \quad p_{\mathrm{l}}+\frac{2 \varepsilon^{2}}{3}\left(\frac{\partial u_{\mathrm{l}}}{\partial x}-2 \frac{\partial v_{\mathrm{l}}}{\partial y}\right)=p_{\mathrm{m}}, \\
c_{\mathrm{l}} & =c_{\mathrm{m}}, \quad \frac{\partial c_{\mathrm{l}}}{\partial y}=\phi_{\mathrm{m}} \frac{\partial c_{\mathrm{m}}}{\partial y} \quad \text { on } y=1 ;
\end{aligned}
$$




$$
\begin{gathered}
\phi_{\mathrm{m}} \kappa_{\mathrm{m}} \frac{\partial p_{\mathrm{m}}}{\partial y}=\phi_{\mathrm{w}} \kappa_{\mathrm{w}} \frac{\partial p_{\mathrm{w}}}{\partial y}, \quad p_{\mathrm{m}}=p_{\mathrm{w}}, \\
c_{\mathrm{m}}=c_{\mathrm{w}}, \quad \phi_{\mathrm{m}} \frac{\partial c_{\mathrm{m}}}{\partial y}=\phi_{\mathrm{w}} \frac{\partial c_{\mathrm{w}}}{\partial y} \quad \text { on } y=1+h_{2} ; \\
u_{\mathrm{f}}=0, \quad-\varepsilon \phi_{\mathrm{w}} \kappa_{\mathrm{w}} \frac{\partial p_{\mathrm{w}}}{\partial y}=v_{\mathrm{f}}, \quad p_{\mathrm{w}}=p_{\mathrm{f}}+\frac{2 \varepsilon^{2}}{3}\left(\frac{\partial u_{\mathrm{f}}}{\partial x}-2 \frac{\partial v_{\mathrm{f}}}{\partial y}\right), \\
c_{\mathrm{w}}=c_{\mathrm{f}}, \quad \phi_{\mathrm{w}} \frac{\partial c_{\mathrm{w}}}{\partial y}=\frac{\partial c_{\mathrm{f}}}{\partial y} \quad \text { on } y=H-h_{4} ; \\
u_{\mathrm{f}}=v_{\mathrm{f}}=0, \quad \frac{\partial c_{\mathrm{f}}}{\partial y}=0 \quad \text { on } y=H .
\end{gathered}
$$

Next we analytically solve for the fluid velocities and pressures at $O(1)$ and $O(\varepsilon)$, before using these expressions to derive equations for the solute concentrations at equivalent orders.

\subsection{FlOW SOLUTION}

To find the leading- and first-order fluid velocities and pressures, we expand all variables in powers of $\varepsilon$, for instance setting $u_{1} \sim u_{l_{0}}+\varepsilon u_{l_{1}}+\varepsilon^{2} u_{l_{2}}+\ldots$, and similarly for the remaining velocities and pressures. At leading order (dropping the subscript 0 ), this yields the following governing equations in the lumen $(0<y<1)$

$$
\frac{\partial u_{1}}{\partial x}+\frac{\partial v_{1}}{\partial y}=0, \quad \frac{\partial^{2} u_{1}}{\partial y^{2}}=\frac{\partial p_{1}}{\partial x}, \quad \frac{\partial p_{1}}{\partial y}=0 ;
$$

in the porous membrane $\left(1<y<1+h_{2}\right)$

$$
u_{\mathrm{m}} \equiv 0, \quad v_{\mathrm{m}}=-\kappa_{\mathrm{m}} \frac{\partial p_{\mathrm{m}}}{\partial y}, \quad \frac{\partial^{2} p_{\mathrm{m}}}{\partial y^{2}}=0 ;
$$

in the cell layer $\left(1+h_{2}<y<H-h_{4}\right)$

$$
u_{\mathrm{w}} \equiv 0, \quad v_{\mathrm{w}}=-\kappa_{\mathrm{w}} \frac{\partial p_{\mathrm{w}}}{\partial y}, \quad \frac{\partial^{2} p_{\mathrm{w}}}{\partial y^{2}}=0 ;
$$

and in the upper fluid layer $\left(H-h_{4}<y<H\right)$

$$
\frac{\partial u_{\mathrm{f}}}{\partial x}+\frac{\partial v_{\mathrm{f}}}{\partial y}=0, \quad \frac{\partial^{2} u_{\mathrm{f}}}{\partial y^{2}}=\frac{\partial p_{\mathrm{f}}}{\partial x}, \quad \frac{\partial p_{\mathrm{f}}}{\partial y}=0 .
$$

At leading order the boundary conditions are given by

$$
\begin{aligned}
& \frac{\partial u_{1}}{\partial y}=0, \quad v_{\mathrm{l}}=0 \quad \text { on } y=0, \\
& u_{\mathrm{l}}=v_{\mathrm{l}}=0, \quad p_{\mathrm{l}}=p_{\mathrm{m}} \quad \text { on } y=1, \\
& \phi_{\mathrm{m}} \kappa_{\mathrm{m}} \frac{\partial p_{\mathrm{m}}}{\partial y}=\phi_{\mathrm{w}} \kappa_{\mathrm{w}} \frac{\partial p_{\mathrm{w}}}{\partial y}, \quad \quad p_{\mathrm{m}}=p_{\mathrm{w}} \quad \text { on } y=1+h_{2}, \\
& u_{\mathrm{f}}=v_{\mathrm{f}}=0, \quad p_{\mathrm{w}}=p_{\mathrm{f}} \quad \text { on } y=H-h_{4}, \\
& u_{\mathrm{f}}=v_{\mathrm{f}}=0 \quad \text { on } y=H .
\end{aligned}
$$

Finally, we impose the up- and down-stream boundary conditions found from matching with the inner solutions in Appendix A.1. Analysis of the fluid equations in the upstream inner region 
shows that fluid flux is conserved in both the lumen and upper fluid layer at $\mathrm{O}(1)$, and so the inlet flux conditions are unchanged (having non-dimensionalised appropriately):

$$
\begin{array}{rlrl}
Q_{1, \text { in }} & =\int_{0}^{1} u_{\mathrm{l}} \mathrm{d} y & \text { at } x=0^{+}, \\
Q_{\mathrm{f}, \text { in }}=\int_{H-h_{4}}^{H} u_{\mathrm{f}} \mathrm{d} y & \text { at } x=0^{+} .
\end{array}
$$

Similarly, analysis of the fluid equations in the downstream inner region shows that the pressure in the lumen and upper fluid layer is constant at $\mathrm{O}(1)$, and so the outlet pressure conditions on the outer solution are unchanged at leading order,

$$
p_{\mathrm{l}}=P_{\mathrm{d}}, \quad p_{\mathrm{f}}=0 \quad \text { at } x=1^{-} .
$$

We are able to solve for the leading-order velocities and pressures analytically, to find in the lumen

$$
u_{\mathrm{l}}=-\frac{3}{2} Q_{1, \mathrm{in}}\left(y^{2}-1\right), \quad v_{\mathrm{l}} \equiv 0, \quad p_{\mathrm{l}}=3 Q_{1, \text { in }}(1-x)+P_{\mathrm{d}}
$$

in the porous membrane

$$
u_{\mathrm{m}} \equiv 0, \quad v_{\mathrm{m}}=-\kappa_{\mathrm{m}} F_{\mathrm{m}}(x), \quad p_{\mathrm{m}}=F_{\mathrm{m}}(x)(y-1)+3 Q_{1, \mathrm{in}}(1-x)+P_{\mathrm{d}} ;
$$

in the cell layer

$$
\begin{gathered}
u_{\mathrm{w}} \equiv 0, \quad v_{\mathrm{w}}=-\frac{\phi_{\mathrm{m}} \kappa_{\mathrm{m}}}{\phi_{\mathrm{w}}} F_{\mathrm{m}}(x), \\
p_{\mathrm{w}}=\frac{\phi_{\mathrm{m}} \kappa_{\mathrm{m}}}{\phi_{\mathrm{w}} \kappa_{\mathrm{w}}}\left(y-\left(1+h_{2}\right)\right) F_{\mathrm{m}}(x)+h_{2} F_{\mathrm{m}}(x)+3 Q_{1, \text { in }}(1-x)+P_{\mathrm{d}}
\end{gathered}
$$

and in the upper fluid layer

$$
u_{\mathrm{f}}=-\frac{6 Q_{\mathrm{f}, \mathrm{in}}}{h_{4}^{3}}(y-H)\left(y-\left(H-h_{4}\right)\right), \quad v_{\mathrm{f}} \equiv 0, \quad p_{\mathrm{f}}=\frac{12 Q_{\mathrm{f}, \mathrm{in}}}{h_{4}^{3}}(1-x),
$$

where for algebraic convenience we have set

$$
\begin{aligned}
F_{\mathrm{m}}(x) & :=A_{\mathrm{m}} x+B_{\mathrm{m}}, \\
A_{\mathrm{m}} & :=-\frac{\phi_{\mathrm{w}} \kappa_{\mathrm{w}}}{\phi_{\mathrm{m}} \kappa_{\mathrm{m}} h_{3}+\phi_{\mathrm{w}} \kappa_{\mathrm{w}} h_{2}}\left(\frac{12 Q_{\mathrm{f}, \mathrm{in}}}{h_{4}^{3}}-3 Q_{1, \mathrm{in}}\right), \\
B_{\mathrm{m}} & :=-A_{\mathrm{m}}-\frac{\phi_{\mathrm{w}} \kappa_{\mathrm{w}} P_{\mathrm{d}}}{\phi_{\mathrm{m}} \kappa_{\mathrm{m}} h_{3}+\phi_{\mathrm{w}} \kappa_{\mathrm{w}} h_{2}} .
\end{aligned}
$$

To determine equations for the solute concentrations up to $\mathrm{O}(\varepsilon)$, we also require the $\mathrm{O}(\varepsilon)$ velocities in the lumen and upper fluid layer. In the lumen, the governing equations at this order are (temporarily returning to subscript 0,1 notation for clarity)

$$
\frac{\partial u_{\mathrm{l}_{1}}}{\partial x}+\frac{\partial v_{\mathrm{l}_{1}}}{\partial y}=0, \quad \frac{\partial p_{\mathrm{l}_{1}}}{\partial y}=0, \quad \frac{\partial^{2} u_{\mathrm{l}_{1}}}{\partial y^{2}}=\frac{\partial p_{\mathrm{l}_{1}}}{\partial x}=0,
$$

subject to

$$
\begin{gathered}
\frac{\partial u_{\mathrm{l}_{1}}}{\partial y}=0, \quad v_{\mathrm{l}_{1}}=0 \quad \text { on } y=0, \\
u_{\mathrm{l}_{1}}=0, \quad v_{\mathrm{l}_{1}}=-\kappa_{\mathrm{m}} \phi_{\mathrm{m}} \frac{\partial p_{\mathrm{m}_{0}}}{\partial y}, \quad p_{\mathrm{l}_{1}}=p_{\mathrm{m}_{1}} \quad \text { on } y=1,
\end{gathered}
$$


and

$$
\int_{0}^{1} u_{\mathrm{l}_{1}} \mathrm{~d} y=0 \quad \text { on } x=0^{+},
$$

where the zero flux condition again follows from the inner region analysis in Appendix A, which shows that fluid flux in the lumen is also conserved at $\mathrm{O}(\varepsilon)$. These can be solved to find

$$
u_{1_{1}}=-\frac{3 \kappa_{\mathrm{m}} \phi_{\mathrm{m}}}{2}\left(\frac{A_{\mathrm{m}}}{2} x^{2}+B_{\mathrm{m}} x\right)\left(y^{2}-1\right), \quad v_{\mathrm{l}_{1}}=\frac{3 \kappa_{\mathrm{m}} \phi_{\mathrm{m}}}{2}\left(A_{\mathrm{m}} x+B_{\mathrm{m}}\right)\left(\frac{y^{3}}{3}-y\right) .
$$

Similarly in the upper fluid layer, the $O(\varepsilon)$ governing equations are

$$
\frac{\partial u_{\mathrm{f}_{1}}}{\partial x}+\frac{\partial v_{\mathrm{f}_{1}}}{\partial y}=0, \quad \frac{\partial p_{\mathrm{f}_{1}}}{\partial y}=0, \quad \frac{\partial^{2} u_{\mathrm{f}_{1}}}{\partial y^{2}}=\frac{\partial p_{\mathrm{f}_{1}}}{\partial x}=0,
$$

with

$$
\begin{gathered}
u_{\mathrm{f}_{1}}=0, \quad v_{\mathrm{f}_{1}}=-\kappa_{\mathrm{w}} \phi_{\mathrm{w}} \frac{\partial p_{\mathrm{w}_{0}}}{\partial y}, \quad p_{\mathrm{w}_{1}}=p_{\mathrm{f}_{1}} \quad \text { on } y=H-h_{4}, \\
u_{\mathrm{f}_{1}}=0, \quad v_{\mathrm{f}_{1}}=0 \quad \text { on } y=H,
\end{gathered}
$$

and

$$
\int_{H-h_{4}}^{H} u_{\mathrm{f}_{1}} \mathrm{~d} y=0 \quad \text { on } x=0^{+} .
$$

Solving these yields

$$
\begin{gathered}
u_{\mathrm{f}_{1}}=\frac{6 \phi_{\mathrm{m}} \kappa_{\mathrm{m}}}{h_{4}^{3}}\left(\frac{A_{\mathrm{m}}}{2} x^{2}+B_{\mathrm{m}} x\right)(y-H)\left(y-\left(H-h_{4}\right)\right) \\
v_{\mathrm{f}_{1}}=-\frac{6 \phi_{\mathrm{m}} \kappa_{\mathrm{m}}}{h_{4}^{3}}\left(A_{\mathrm{m}} x+B_{\mathrm{m}}\right)\left(\frac{y^{3}}{3}-\frac{1}{2}\left(2 H-h_{4}\right) y^{2}+H\left(H-h_{4}\right) y-H^{2}\left(\frac{H}{3}-\frac{h_{4}}{2}\right)\right) .
\end{gathered}
$$

\subsection{MASS TRANSPORT}

Now we return to the governing equations for the solute in (19)-(22), expanding the concentration in each section in powers of $\varepsilon$ as in $\S 3.5$. At leading order (dropping the subscript 0 ) we find that diffusion in the $y$-direction dominates throughout, so that

$$
\frac{\partial^{2} c_{i}}{\partial y^{2}}=0, \quad i=1, \mathrm{~m}, \mathrm{w}, \mathrm{f},
$$

with the corresponding boundary conditions

$$
\begin{aligned}
& \frac{\partial c_{1}}{\partial y}=0 \quad \text { on } y=0, \\
& c_{1}=c_{\mathrm{m}}, \quad \frac{\partial c_{1}}{\partial y}=\phi_{\mathrm{m}} \frac{\partial c_{\mathrm{m}}}{\partial y} \quad \text { on } y=1, \\
& c_{\mathrm{m}}=c_{\mathrm{w}}, \quad \phi_{\mathrm{m}} \frac{\partial c_{\mathrm{m}}}{\partial y}=\phi_{\mathrm{w}} \frac{\partial c_{\mathrm{w}}}{\partial y} \quad \text { on } y=1+h_{2}, \\
& c_{\mathrm{w}}=c_{\mathrm{f}}, \quad \phi_{\mathrm{w}} \frac{\partial c_{\mathrm{w}}}{\partial y}=\frac{\partial c_{\mathrm{f}}}{\partial y} \quad \text { on } y=H-h_{4}, \\
& \frac{\partial c_{\mathrm{f}}}{\partial y}=0 \quad \text { on } y=H .
\end{aligned}
$$


Solving this system implies that (as in the lower Pe regime of [5]) at leading order the concentration is independent of $y$ and hence common to all regions, that is, $c_{i}=c_{0}(x, t)(i=1, \mathrm{~m}, \mathrm{w}, \mathrm{f})$. Hence the solute concentration must be independent of $y$ at leading order as it leaves the upstream inner region in order to match with this outer solution. We denote this limit of the inner concentration by $c_{\text {in }}(t)$ and, given the conservation of fluid flux across this inner region, we have

$$
c_{0}(0, t)=c_{\mathrm{in}}(t)=\frac{Q_{\mathrm{l}, \mathrm{in}} c_{\mathrm{l}, \mathrm{in}}(t)+Q_{\mathrm{f}, \text { in }} c_{\mathrm{f}, \text { in }}(t)}{Q_{\mathrm{l}, \mathrm{in}}+Q_{\mathrm{f}, \mathrm{in}}} \quad \text { at } x=0^{+} .
$$

Downstream, we find that the concentration in the inner region is a function of $t$ only (see Appendix A.1), and will be determined by the value of $c_{0}$ as $x \rightarrow 1$.

To determine the leading-order concentration $c_{0}$ we must consider the $\mathrm{O}(\varepsilon)$ system. In doing so we will also be able to find the next order correction to the solute concentration in each section up to an unknown function of $x$ and $t$, which would need to be determined by considering the $\mathrm{O}\left(\varepsilon^{2}\right)$ set of equations. In the flow regime considered, these correction terms could become significant for high values of $\mathrm{Pe}$ as advection plays a larger role in the leading-order concentration field. In the following, we again return to subscript 0,1 notation for clarity.

Equating coefficients of $\varepsilon$ in each section, with $\varepsilon \mathrm{Pe}=\mathrm{O}(1)$ as $\varepsilon \rightarrow 0$, yields

$$
\begin{gathered}
\varepsilon \operatorname{Pe}\left(\frac{\partial c_{0}}{\partial t}+\frac{3}{2} Q_{1, \text { in }}\left(1-y^{2}\right) \frac{\partial c_{0}}{\partial x}\right)=\frac{\partial^{2} c_{l_{1}}}{\partial y^{2}}, \quad \varepsilon \operatorname{Pe} \frac{\partial c_{0}}{\partial t}=\frac{\partial^{2} c_{\mathrm{m}_{1}}}{\partial y^{2}}, \\
\varepsilon \operatorname{Pe} \frac{\partial c_{0}}{\partial t}=\frac{\partial^{2} c_{\mathrm{w}_{1}}}{\partial y^{2}}-\frac{\mathcal{R}}{\phi_{\mathrm{w}}}, \quad \varepsilon \operatorname{Pe}\left(\frac{\partial c_{0}}{\partial t}+\frac{6}{h_{4}^{3}} Q_{\mathrm{f}, \mathrm{in}}(y-H)\left(H-h_{4}-y\right) \frac{\partial c_{0}}{\partial x}\right)=\frac{\partial^{2} c_{\mathrm{f}_{1}}}{\partial y^{2}},
\end{gathered}
$$

where we have also substituted in the known forms for $\mathbf{u}_{\mathrm{l}_{0}}$ and $\mathbf{u}_{\mathrm{f}_{0}}$ from (40) and (43), respectively. The boundary conditions at this order are

$$
\begin{aligned}
& \frac{\partial c_{l_{1}}}{\partial y}=0 \quad \text { on } y=0, \\
& c_{\mathrm{l}_{1}}=c_{\mathrm{m}_{1}}, \quad \frac{\partial c_{\mathrm{l}_{1}}}{\partial y}=\phi_{\mathrm{m}} \frac{\partial c_{\mathrm{m}_{1}}}{\partial y} \quad \text { on } y=1 \text {, } \\
& c_{\mathrm{m}_{1}}=c_{\mathrm{w}_{1}}, \quad \phi_{\mathrm{m}} \frac{\partial c_{\mathrm{m}_{1}}}{\partial y}=\phi_{\mathrm{w}} \frac{\partial c_{\mathrm{w}_{1}}}{\partial y} \quad \text { on } y=1+h_{2}, \\
& c_{\mathrm{w}_{1}}=c_{\mathrm{f}_{1}}, \quad \phi_{\mathrm{w}} \frac{\partial c_{\mathrm{w}_{1}}}{\partial y}=\frac{\partial c_{\mathrm{f}_{1}}}{\partial y} \quad \text { on } y=H-h_{4}, \\
& \frac{\partial c_{\mathrm{f}_{1}}}{\partial y}=0 \quad \text { on } y=H .
\end{aligned}
$$

Beginning in the lumen, the system (60)-(65) can be solved to find

$$
\begin{aligned}
c_{\mathrm{l}_{1}}= & \varepsilon \operatorname{Pe}\left(\frac{y^{2}}{2} \frac{\partial c_{0}}{\partial t}+\frac{Q_{1, \text { in }}}{8}\left(6 y^{2}-y^{4}\right) \frac{\partial c_{0}}{\partial x}+g(x, t)\right) \\
c_{\mathrm{m}_{1}}= & \varepsilon \operatorname{Pe}\left(\left(\frac{y^{2}}{2}+\left(\frac{1}{\phi_{\mathrm{m}}}-1\right)(y-1)\right) \frac{\partial c_{0}}{\partial t}+Q_{1, \text { in }}\left(\frac{y-1}{\phi_{\mathrm{m}}}+\frac{5}{8}\right) \frac{\partial c_{0}}{\partial x}+g(x, t)\right) \\
c_{\mathrm{w}_{1}}= & \varepsilon \operatorname{Pe}\left(A_{\mathrm{w}}(y) \frac{\partial c_{0}}{\partial t}+Q_{1, \mathrm{in}}\left(\frac{y-\left(1+h_{2}\right)}{\phi_{\mathrm{w}}}+\frac{h_{2}}{\phi_{\mathrm{m}}}+\frac{5}{8}\right) \frac{\partial c_{0}}{\partial x}\right. \\
& \left.\quad+\frac{\mathcal{R}}{2 \varepsilon \operatorname{Pe} \phi_{\mathrm{w}}}\left(1+h_{2}-y\right)^{2}+g(x, t)\right) \\
c_{\mathrm{f}_{1}}= & \varepsilon \operatorname{Pe}\left(A_{\mathrm{f}}(y) \frac{\partial c_{0}}{\partial t}+\frac{Q_{\mathrm{f}, \text { in }}}{h_{4}^{3}} B_{\mathrm{f}}(y) \frac{\partial c_{0}}{\partial x}+Q_{1, \mathrm{in}}\left(\frac{h_{2}}{\phi_{\mathrm{m}}}+\frac{h_{3}}{\phi_{\mathrm{w}}}+\frac{5}{8}\right) \frac{\partial c_{0}}{\partial x}+\frac{h_{3}^{2} \mathcal{R}}{2 \varepsilon \operatorname{Pe} \phi_{\mathrm{w}}}+g(x, t)\right)
\end{aligned}
$$


for some unknown function $g(x, t)$ to be determined from the $\mathrm{O}\left(\varepsilon^{2}\right)$ solvability condition, where

$$
\begin{aligned}
A_{\mathrm{w}}(y) & :=\frac{y^{2}}{2}-\left(1+h_{2}\right) y+\left(1+h_{2}\right)^{2}+\frac{1+h_{2} \phi_{\mathrm{m}}}{\phi_{\mathrm{w}}}\left(y-\left(1+h_{2}\right)\right)+h_{2}\left(\frac{1}{\phi_{\mathrm{m}}}-1\right), \\
A_{\mathrm{f}}(y) & :=\frac{y^{2}}{2}-H y+\frac{1}{2}\left(H^{2}-h_{4}^{2}+h_{3}^{2}+\left(1+h_{2}\right)^{2}\right)+\frac{h_{3}\left(1+h_{2} \phi_{\mathrm{m}}\right)}{\phi_{\mathrm{w}}}+h_{2}\left(\frac{1}{\phi_{\mathrm{m}}}-1\right), \\
B_{\mathrm{f}}(y) & :=-\frac{y^{4}}{2}+\left(2 H-h_{4}\right) y^{3}-3 H\left(H-h_{4}\right) y^{2}-H^{2}\left(3 h_{4}-2 H\right) y-\frac{H^{4}}{2}+h_{4} H^{3}-\frac{h_{4}^{4}}{2} .
\end{aligned}
$$

Furthermore, in applying the continuity of flux condition from (64) on $y=H-h_{4}$, we also obtain the following solvability condition for $c_{0}$ :

$$
\bar{h} \frac{\partial c_{0}}{\partial t}+\left(Q_{1, \text { in }}+Q_{\mathrm{f}, \text { in }}\right) \frac{\partial c_{0}}{\partial x}=-\frac{h_{3} \mathcal{R}}{\varepsilon \mathrm{Pe}},
$$

subject to

$$
c_{0}=c_{\text {in }} \quad \text { at } x=0^{+},
$$

and where

$$
\bar{h}=1+h_{2} \phi_{\mathrm{m}}+h_{3} \phi_{\mathrm{w}}+h_{4} .
$$

To find $g(x, t)$, we consider the solute equations from (19)-(22) at $\mathrm{O}\left(\varepsilon^{2}\right)$ in each section, which are

$$
\begin{aligned}
\varepsilon \operatorname{Pe}\left(\frac{\partial c_{\mathrm{l}_{1}}}{\partial t}+\frac{\partial}{\partial x}\left(c_{0} u_{\mathrm{l}_{1}}+c_{\mathrm{l}_{1}} u_{\mathrm{l}_{0}}\right)+\frac{\partial}{\partial y}\left(c_{0} v_{\mathrm{l}_{1}}\right)\right) & =\frac{\partial^{2} c_{0}}{\partial x^{2}}+\frac{\partial^{2} c_{\mathrm{l}_{2}}}{\partial y^{2}} \\
\varepsilon \operatorname{Pe} \frac{\partial c_{\mathrm{m}_{1}}}{\partial t} & =\frac{\partial^{2} c_{0}}{\partial x^{2}}+\frac{\partial^{2} c_{\mathrm{m}_{2}}}{\partial y^{2}} \\
\varepsilon \operatorname{Pe} \frac{\partial c_{\mathrm{w}_{1}}}{\partial t} & =\frac{\partial^{2} c_{0}}{\partial x^{2}}+\frac{\partial^{2} c_{\mathrm{w}_{2}}}{\partial y^{2}} \\
\varepsilon \operatorname{Pe}\left(\frac{\partial c_{\mathrm{f}_{1}}}{\partial t}+\frac{\partial}{\partial x}\left(c_{0} u_{\mathrm{f}_{1}}+c_{\mathrm{f}_{1}} u_{\mathrm{f}_{0}}\right)+\frac{\partial}{\partial y}\left(c_{0} v_{\mathrm{f}_{1}}\right)\right) & =\frac{\partial^{2} c_{0}}{\partial x^{2}}+\frac{\partial^{2} c_{\mathrm{f}_{2}}}{\partial y^{2}}
\end{aligned}
$$

Integrating equations (76) and (79) with respect to $y$ across the lumen and upper fluid layer respectively, and substituting in the known forms for the leading- and first-order variables from (40), (43), (48), (52), (66)-(69), yields

$$
(\varepsilon \mathrm{Pe})^{2}\left(\frac{\alpha_{1}}{\varepsilon \mathrm{Pe}} \frac{\partial c_{0}}{\partial x}+\alpha_{2} \frac{\partial^{2} c_{0}}{\partial x \partial t}+\alpha_{3} \frac{\partial^{2} c_{0}}{\partial t^{2}}+\alpha_{4} \frac{\partial^{2} c_{0}}{\partial x^{2}}+\frac{\partial g}{\partial t}+Q_{1, \text { in }} \frac{\partial g}{\partial x}\right)=\frac{\partial^{2} c_{0}}{\partial x^{2}}+\left.\frac{\partial c_{\mathrm{l}_{2}}}{\partial y}\right|_{y=1},
$$

and

$$
\begin{aligned}
(\varepsilon \mathrm{Pe})^{2}\left(\frac{\beta_{1}}{\varepsilon \operatorname{Pe}} \frac{\partial c_{0}}{\partial x}+\beta_{2} \frac{\partial^{2} c_{0}}{\partial x \partial t}+\beta_{3} \frac{\partial^{2} c_{0}}{\partial t^{2}}+\beta_{4} \frac{\partial^{2} c_{0}}{\partial x^{2}}+h_{4} \frac{\partial g}{\partial t}+Q_{\mathrm{f}, \mathrm{in}} \frac{\partial g}{\partial x}\right) & = \\
& h_{4} \frac{\partial^{2} c_{0}}{\partial x^{2}}-\left.\frac{\partial c_{\mathrm{f}_{2}}}{\partial y}\right|_{y=H-h_{4}}
\end{aligned}
$$

where

$$
\alpha_{1}=\kappa_{\mathrm{m}} \phi_{\mathrm{m}}\left(\frac{A_{\mathrm{m}}}{2} x^{2}+B_{\mathrm{m}} x\right), \quad \alpha_{2}=\frac{13}{40} Q_{1, \mathrm{in}}, \quad \alpha_{3}=\frac{1}{6}, \quad \alpha_{4}=\frac{39}{280} Q_{1, \mathrm{in}}^{2},
$$


and

$$
\begin{aligned}
& \beta_{1}=-\alpha_{1}, \\
& \beta_{2}=Q_{\mathrm{f}, \text { in }}\left(\frac{1+h_{2}^{2}+h_{3}^{2}}{2}+\frac{h_{2}}{\phi_{\mathrm{m}}}+\frac{h_{3}}{\phi_{\mathrm{w}}}+\frac{h_{2} h_{3} \phi_{\mathrm{m}}}{\phi_{\mathrm{w}}}-\frac{7 h_{4}^{2}}{10}\right)+Q_{1, \text { in }} h_{4}\left(\frac{h_{2}}{\phi_{\mathrm{m}}}+\frac{h_{3}}{\phi_{\mathrm{w}}}+\frac{5}{8}\right), \\
& \beta_{3}=h_{4}\left(\frac{1+h_{2}^{2}+h_{3}^{2}}{2}+\frac{h_{2}}{\phi_{\mathrm{m}}}+\frac{h_{3}}{\phi_{\mathrm{w}}}-\frac{h_{4}^{2}}{3}+\frac{h_{2} h_{3} \phi_{\mathrm{m}}}{\phi_{\mathrm{w}}}\right), \\
& \beta_{4}=Q_{\mathrm{f}, \text { in }}\left(Q_{\mathrm{l}, \text { in }}\left(\frac{h_{2}}{\phi_{\mathrm{m}}}+\frac{h_{3}}{\phi_{\mathrm{w}}}+\frac{5}{8}\right)-\frac{13 h_{4} Q_{\mathrm{f}, \text { in }}}{35}\right) .
\end{aligned}
$$

In order to eliminate $\partial c_{l_{2}} /\left.\partial y\right|_{y=1}$ and $\partial c_{\mathrm{f}_{2}} /\left.\partial y\right|_{y=H-h_{4}}$, we can use equations (77) and (78) for the evolution of $c_{\mathrm{m}_{2}}$ and $c_{\mathrm{w}_{2}}$, respectively, and the following $\mathrm{O}\left(\varepsilon^{2}\right)$ boundary conditions

$$
\begin{aligned}
\frac{\partial c_{\mathrm{l}_{2}}}{\partial y} & =\phi_{\mathrm{m}} \frac{\partial c_{\mathrm{m}_{2}}}{\partial y} & & \text { on } y=1, \\
\phi_{\mathrm{m}} \frac{\partial c_{\mathrm{m}_{2}}}{\partial y} & =\phi_{\mathrm{w}} \frac{\partial c_{\mathrm{w}_{2}}}{\partial y} & & \text { on } y=1+h_{2}, \\
\phi_{\mathrm{w}} \frac{\partial c_{\mathrm{w}_{2}}}{\partial y} & =\frac{\partial c_{\mathrm{f}_{2}}}{\partial y} & & \text { on } y=H-h_{4},
\end{aligned}
$$

to find that

$$
\begin{aligned}
\left.\frac{\partial c_{\mathrm{l}_{2}}}{\partial y}\right|_{y=1}=(\varepsilon \mathrm{Pe})^{2}\left(\gamma_{2} \frac{\partial^{2} c_{0}}{\partial x \partial t}+\gamma_{3} \frac{\partial^{2} c_{0}}{\partial t^{2}}\right. & \left.-\left(h_{2} \phi_{\mathrm{m}}+h_{3} \phi_{\mathrm{w}}\right) \frac{\partial g}{\partial t}\right) \\
& +\left(h_{2} \phi_{\mathrm{m}}+h_{3} \phi_{\mathrm{w}}\right) \frac{\partial^{2} c_{0}}{\partial x^{2}}+\left.\frac{\partial c_{\mathrm{f}_{2}}}{\partial y}\right|_{y=H-h_{4}},
\end{aligned}
$$

where

$$
\begin{aligned}
& \gamma_{2}=-Q_{1, \text { in }}\left(\frac{15}{24}\left(h_{2} \phi_{\mathrm{m}}+h_{3} \phi_{\mathrm{w}}\right)+\frac{h_{2}^{2}+h_{3}^{2}}{2}+\frac{h_{2} h_{3} \phi_{\mathrm{w}}}{\phi_{\mathrm{m}}}\right), \\
& \gamma_{3}=-\left(\frac{\left(1+h_{2}^{2}+h_{3}^{2}\right)\left(1+h_{2} \phi_{\mathrm{m}}+h_{3} \phi_{\mathrm{w}}\right)}{2}-\frac{1}{2}-\frac{h_{2}^{3} \phi_{\mathrm{m}}+h_{3}^{3} \phi_{\mathrm{w}}}{3}+\frac{h_{2} h_{3} \phi_{\mathrm{w}}}{\phi_{\mathrm{m}}}\right) .
\end{aligned}
$$

Hence, by adding together (80) and (81) we can obtain the following solvability condition for $g(x, t)$ :

$$
\begin{aligned}
& \bar{h} \frac{\partial g}{\partial t}+\left(Q_{1, \text { in }}+Q_{\mathrm{f}, \text { in }}\right) \frac{\partial g}{\partial x}= \\
& \quad\left(\gamma_{2}-\alpha_{2}-\beta_{2}\right) \frac{\partial^{2} c_{0}}{\partial x \partial t}+\left(\gamma_{3}-\alpha_{3}-\beta_{3}\right) \frac{\partial^{2} c_{0}}{\partial t^{2}}+\left(\frac{\bar{h}}{(\varepsilon \mathrm{Pe})^{2}}-\alpha_{4}-\beta_{4}\right) \frac{\partial^{2} c_{0}}{\partial x^{2}} .
\end{aligned}
$$

However, the physical interpretation of $g(x, t)$ is not immediately clear. To formulate this system in a more physically relevant way, we may rewrite equation (89) in terms of the globally averaged concentration at $\mathrm{O}(\varepsilon)$, which we denote by $\bar{c}_{1}$. To determine $\bar{c}_{1}$, we average the $\mathrm{O}(\varepsilon)$ concentrations in each section in turn, and then add them together, with appropriate weighting in the membrane and cell layer:

$$
\bar{c}_{1}=\bar{c}_{\mathrm{l}_{1}}+h_{2} \phi_{\mathrm{m}} \bar{c}_{\mathrm{m}_{1}}+h_{3} \phi_{\mathrm{w}} \bar{c}_{\mathrm{w}_{1}}+h_{4} \bar{c}_{\mathrm{f}_{1}}
$$

where

$$
\begin{gathered}
\bar{c}_{\mathrm{l}_{1}}=\int_{0}^{1} c_{\mathrm{l}_{1}} \mathrm{~d} y, \quad \bar{c}_{\mathrm{m}_{1}}=\frac{1}{h_{2}} \int_{1}^{1+h_{2}} c_{\mathrm{m}_{1}} \mathrm{~d} y, \\
\bar{c}_{\mathrm{w}_{1}}=\frac{1}{h_{3}} \int_{1+h_{2}}^{1+h_{2}+h_{3}} c_{\mathrm{w}_{1}} \mathrm{~d} y, \quad \bar{c}_{\mathrm{f}_{1}}=\frac{1}{h_{4}} \int_{H-h_{4}}^{H} c_{\mathrm{f}_{1}} \mathrm{~d} y .
\end{gathered}
$$


Using the expressions for the $\mathrm{O}(\varepsilon)$ concentrations from equations (66)-(69), we find

$$
\bar{c}_{1}=\varepsilon \operatorname{Pe}\left(\lambda_{1} \frac{\partial c_{0}}{\partial t}+\lambda_{2} \frac{\partial c_{0}}{\partial x}+\frac{h_{3}^{2}\left(h_{3} \phi_{\mathrm{w}}+3 h_{4}\right) \mathcal{R}}{6 \varepsilon \operatorname{Pe} \phi_{\mathrm{w}}}+\bar{h} g(x, t)\right)
$$

where

$$
\begin{aligned}
& \lambda_{1}=\frac{1}{2}\left(1+h_{2}^{2}+h_{3}^{2}\right) \bar{h}-\frac{1+h_{2}^{3} \phi_{\mathrm{m}}+h_{3}^{3} \phi_{\mathrm{w}}+h_{4}^{3}}{3} \\
&+ h_{4}\left(\frac{h_{2}}{\phi_{\mathrm{m}}}+\frac{h_{3}}{\phi_{\mathrm{w}}}\right)+\frac{h_{2} h_{3} \phi_{\mathrm{w}}}{\phi_{\mathrm{m}}}+\frac{h_{2} h_{3} h_{4} \phi_{\mathrm{m}}}{\phi_{\mathrm{w}}}, \\
& \lambda_{2}=Q_{1, \text { in }}\left(\frac{1}{2}\left(h_{2}^{2}+h_{3}^{2}\right)+\frac{5}{8}\left(h_{2} \phi_{\mathrm{m}}+h_{3} \phi_{\mathrm{w}}+h_{4}\right)\right. \\
&\left.+\frac{h_{2} h_{3} \phi_{\mathrm{w}}}{\phi_{\mathrm{m}}}+h_{4}\left(\frac{h_{2}}{\phi_{\mathrm{m}}}+\frac{h_{3}}{\phi_{\mathrm{w}}}\right)+\frac{9}{40}\right)-\frac{7 h_{4}^{2} Q_{\mathrm{f}, \text { in }}}{20} .
\end{aligned}
$$

We can therefore rewrite equation (89) for $g(x, t)$ in terms of $\bar{c}_{1}$, which yields

$$
\varepsilon \operatorname{Pe}\left(\bar{h} \frac{\partial \bar{c}_{1}}{\partial t}+\left(Q_{1, \text { in }}+Q_{\mathrm{f}, \text { in }}\right) \frac{\partial \bar{c}_{1}}{\partial x}\right)=K \frac{\partial^{2} c_{0}}{\partial x^{2}},
$$

where we have used the fact that differentiating equation (73) with respect to $x$ yields the relationship

$$
\frac{\partial^{2} c_{0}}{\partial x \partial t}=-\frac{Q_{1, \text { in }}+Q_{\mathrm{f}, \text { in }}}{\bar{h}} \frac{\partial^{2} c_{0}}{\partial x^{2}}
$$

in order to eliminate $\partial^{2} c_{0} / \partial x \partial t$. We also note that the coefficients of $\partial^{2} c_{0} / \partial t^{2}$ cancel, so that this term does not appear in the final equation. The constant $K$ can be expressed in terms of previous constants as

$$
K=\bar{h}^{2}\left(1+(\varepsilon \mathrm{Pe})^{2} \widetilde{K}\right)
$$

where the $\mathrm{O}(1)$ constant $\widetilde{K}$ is given by

$$
\widetilde{K}=\frac{1}{\bar{h}^{2}}\left(\left(Q_{1, \text { in }}+Q_{\mathrm{f}, \text { in }}\right)\left(\alpha_{2}+\beta_{2}-\gamma_{2}\right)-\frac{\left(Q_{1, \text { in }}+Q_{\mathrm{f}, \text { in }}\right)^{2} \lambda_{1}}{\bar{h}}-\bar{h}\left(\alpha_{4}+\beta_{4}\right)\right) .
$$

We now again consider the upstream inner region, and through the analysis in Appendix A.1 find that there is an $\mathrm{O}(\varepsilon)$ contribution to the outer inlet concentration, due to uptake of solute in the inner region. This will affect the outer solution in the form of a boundary condition on $\bar{c}_{1}$ at $x=0^{+}$and is an unknown function of $t$ which would need to be determined by numerically solving the full inner problem. This would be extremely computationally expensive due to the complex geometry of the ECS ports and lies beyond the scope of this paper. Instead, we denote this degree of freedom by $\bar{h} \bar{c}_{\mathrm{u}}(t)$ (we have introduced $\bar{h}$ for algebraic convenience later on) and so the appropriate upstream boundary condition to apply on $\bar{c}_{1}$ is

$$
\bar{c}_{1}\left(0^{+}, t\right)=\bar{h} \bar{c}_{\mathrm{u}}(t) .
$$

This then fully determines the solute concentrations at this order and hence again no condition needs to be applied downstream, with the outer solution instead influencing the $O(\varepsilon)$ inner solution. 
Through equations (73) and (95), and boundary conditions (74) and (99), we therefore have the following system to solve for $c_{0}$ and $\bar{c}_{1}$ :

$$
\begin{gathered}
\bar{h} \frac{\partial c_{0}}{\partial t}+\left(Q_{1, \text { in }}+Q_{\mathrm{f}, \text { in }}\right) \frac{\partial c_{0}}{\partial x}=-\frac{h_{3} \mathcal{R}}{\varepsilon \mathrm{Pe}}, \\
\varepsilon \operatorname{Pe}\left(\bar{h} \frac{\partial \bar{c}_{1}}{\partial t}+\left(Q_{1, \text { in }}+Q_{\mathrm{f}, \text { in }}\right) \frac{\partial \bar{c}_{1}}{\partial x}\right)=\bar{h}^{2}\left(1+(\varepsilon \mathrm{Pe})^{2} \widetilde{K}\right) \frac{\partial^{2} c_{0}}{\partial x^{2}}, \\
c_{0}=c_{\text {in }}(t), \quad \bar{c}_{1}=\bar{h} \bar{c}_{\mathrm{u}}(t) \quad \text { at } x=0^{+},
\end{gathered}
$$

given an appropriate form for $c_{\text {in }}(t)$.

\subsection{Constant inlet concentration}

We first consider the simplest case in which $c_{\text {in }}$ is constant in time, i.e. the lumen inlet is maintained at constant concentration throughout the experiment. In this case we can use the method of characteristics to solve (73) and (95) subject to boundary conditions (74) and (99), and the initial conditions $c_{0}(x, 0)=\bar{c}_{1}(x, 0)=0$. Analysis of the inner solution (see Appendix A.1) shows that $\bar{c}_{\mathrm{u}}$ (and so, in steady-state, $\bar{c}_{1}$ ) is a constant, independent of time. This yields

$$
\begin{aligned}
& c_{0}= \begin{cases}-\frac{h_{3} \mathcal{R}}{\varepsilon \operatorname{Pe}\left(Q_{1, \text { in }}+Q_{\mathrm{f}, \mathrm{in}}\right)} x+c_{\mathrm{in}} & \text { for } 0 \leq x \leq \xi, \\
0 & \text { otherwise, }\end{cases} \\
& \bar{c}_{1}= \begin{cases}\bar{h} \bar{c}_{\mathrm{u}} & \text { for } 0 \leq x \leq \xi, \\
0 & \text { otherwise },\end{cases}
\end{aligned}
$$

where

$$
\xi=\min \left(\frac{Q_{1, \text { in }}+Q_{\mathrm{f}, \text { in }}}{\bar{h}} t, \frac{\varepsilon \operatorname{Pe}\left(Q_{1, \text { in }}+Q_{\mathrm{f}, \text { in }}\right) c_{\text {in }}}{h_{3} \mathcal{R}}, 1\right) .
$$

The first term in the definition of $\xi$ denotes the $x$ coordinate that the wave of solute has reached by time $t$, whilst the second term represents the $x$ value at which the concentration reaches 0 due to uptake. Thus, as expected, constant uptake of solute in the cell layer results in a linear concentration profile at leading order.

\subsection{Averaged formulation}

To investigate numerically unsteady results arising from situations in which $c_{\text {in }}$ is not constant, and motivated by similar problems in the literature (as discussed in $\S 1$ ), we return to equations (73) and (95) along with boundary conditions

$$
c_{0}=c_{\mathrm{in}}(t), \quad \bar{c}_{1}=\left(1+\phi_{\mathrm{m}} h_{2}+\phi_{\mathrm{w}} h_{3}+h_{4}\right) \bar{c}_{\mathrm{u}}(t) \quad \text { at } x=0^{+},
$$

and initial conditions

$$
c_{0}=0, \quad \bar{c}_{1}=0 \quad \text { at } t=0 .
$$

By setting

$$
\bar{c}_{0}=\bar{h} c_{0},
$$

we can combine equations (73) and (95) to yield

$$
\frac{\partial \bar{c}}{\partial t}+\frac{Q_{1, \text { in }}+Q_{\mathrm{f}, \mathrm{in}}}{\bar{h}} \frac{\partial \bar{c}}{\partial x}=D_{\mathrm{eff}} \frac{\partial^{2} \bar{c}}{\partial x^{2}}-\frac{h_{3} \mathcal{R}}{\varepsilon \mathrm{Pe}},
$$

where

$$
\bar{c}=\bar{c}_{0}+\varepsilon \bar{c}_{1}
$$


and

$$
D_{\text {eff }}=\frac{1}{\mathrm{Pe}}\left(1+(\varepsilon \mathrm{Pe})^{2} \widetilde{K}\right),
$$

where $\widetilde{K}$ is defined in equation (98). Equation (107) describes the transport of the weighted, averaged solute concentration $\bar{c}$ throughout the bioreactor, accurate up to and including terms of $\mathrm{O}(\varepsilon)$. If we consider the form of $D_{\text {eff }}$ we recognise it as an effective diffusion coefficient (of $\mathrm{O}(1 / \mathrm{Pe})=\mathrm{O}(\varepsilon))$ of a similar form to that found in classical Taylor dispersion [9-11], illustrating the fact that the diffusion of the (averaged) solute throughout the bioreactor is enhanced by the flow field. In $\S 4.2 .1$ we will investigate the dependence of this enhanced diffusion on relevant dimensionless parameters in our model.

We note that in combining the $\mathrm{O}(1)$ and $\mathrm{O}(\varepsilon)$ equations we have, through the addition of a diffusion term at leading order, moved from a coupled system of two hyperbolic PDEs with the necessary two upstream boundary conditions to a single, parabolic PDE with a single boundary condition:

$$
\bar{c}=\bar{h}\left(c_{\mathrm{in}}(t)+\varepsilon c_{\mathrm{u}}(t)\right) \quad \text { at } \quad x=0^{+} .
$$

The parabolic nature of the system will be advantageous for solving the problem numerically; however, in order to do so we must impose a boundary condition at $x=1$. The exact choice of boundary condition is unimportant, as long as we are not introducing significant error through this averaged formulation. Any such error will become apparent in a boundary layer near $x=1$ in which the diffusion term is no longer smaller than the remaining terms in equation (107) and instead there is a dominant balance between advection and diffusion. From analysis of this boundary layer (see Appendix B), we find that choosing the condition

$$
\frac{\partial \bar{c}}{\partial x}=0 \quad \text { at } \quad x=1
$$

results in an error of $\mathrm{O}\left(\varepsilon^{2}\right)$ away from this boundary layer, and of $\mathrm{O}(\varepsilon)$ within the boundary layer. Hence, except for in a region of width $\mathrm{O}(\varepsilon)$ near $x=1$ the errors introduced only come in at orders greater than the accuracy of the system, and thus this is an appropriate choice for the second boundary condition to impose on (107).

In the limit of an impermeable membrane and cell layer $\left(\phi_{\mathrm{m}}, \phi_{\mathrm{w}} \rightarrow 0\right)$, we confirm that we recover from our asymptotic analysis the equations of classical Taylor dispersion in a pipe. Upon averaging the resulting equations in the lumen and upper fluid layer at $\mathrm{O}(1)$ and $\mathrm{O}(\varepsilon)$, combining orders and redimensionalising (details of which we omit for brevity), we find

$$
\begin{aligned}
\frac{\partial \bar{c}_{1}}{\partial t}+\bar{u}_{1} \frac{\partial \bar{c}_{\mathrm{l}}}{\partial x} & =D\left(1+\frac{1}{210} \frac{\left(2 h_{1}\right)^{2} \bar{u}_{1}^{2}}{D^{2}}\right) \frac{\partial^{2} \bar{c}_{1}}{\partial x^{2}}, \\
\frac{\partial \bar{c}_{\mathrm{f}}}{\partial t}+\bar{u}_{\mathrm{f}} \frac{\partial \bar{c}_{\mathrm{f}}}{\partial x} & =D\left(1+\frac{1}{210} \frac{\left(h_{4}\right)^{2} \bar{u}_{\mathrm{f}}^{2}}{D^{2}}\right) \frac{\partial^{2} \bar{c}_{\mathrm{f}}}{\partial x^{2}} .
\end{aligned}
$$

We note that the mean velocity in the lumen is the same whether or not we consider the halflumen $0<y<h_{1}$ or the full lumen $-h_{1}<y<h_{1}$. Hence the result for the half-lumen problem considered here is equivalent to that for the full lumen, only valid for a different range of $y$ values. The classical Taylor dispersion result corresponds to Poiseuille flow in a full channel of width $2 h_{1}$, and hence here we must consider the full lumen for the purposes of comparison. The classical Taylor dispersion result for flow in a two-dimensional channel of width $d$ and mean velocity $U$ is $[10,23]$

$$
\frac{\partial c}{\partial t}+U \frac{\partial c}{\partial x}=D\left(1+\frac{U^{2} d^{2}}{210 D^{2}}\right) \frac{\partial^{2} c}{\partial x^{2}}
$$

and thus equation (112) agrees with the classical result for the (full) lumen (width $2 h_{1}$, mean velocity $Q_{1, \text { in }} / h_{1}$ ) and equation (113) with the classical result for the upper fluid layer (width $h_{4}$, mean velocity $\left.Q_{\mathrm{f}, \text { in }} / h_{4}\right)$. 


\section{Results}

In the following we investigate the behaviour of the solutions obtained in $\S \S 3.7,3.8$, focussing on how variations in key parameter values affect the distribution and uptake of solute throughout the outer region.

\subsection{Constant inlet concentration}

We first consider the solutions to the outer problem derived in $\S 3.6$ at both $O(1)$ and $O(\varepsilon)$ and investigate the qualitative trends arising from varying certain key parameters. We now focus on the steady-state solutions (i.e. once the initial wave of solute has passed through the bioreactor) and recall from (101)-(103) that we have

$$
c_{0}= \begin{cases}-\frac{h_{3} \mathcal{R}}{\varepsilon \operatorname{Pe}\left(Q_{1, \text { in }}+Q_{\mathrm{f}, \text { in }}\right)} x+c_{\text {in }} & \text { for } 0 \leq x \leq \min \left(\frac{\varepsilon \operatorname{Pe}\left(Q_{1, \text { in }}+Q_{\mathrm{f}, \text { in }}\right) c_{\mathrm{in}}}{h_{3} \mathcal{R}}, 1\right), \\ 0 & \text { otherwise. }\end{cases}
$$

Thus at leading order, the nutrient concentration decreases linearly in $x$, and provided

$$
c_{\text {in }}>\frac{h_{3} \mathcal{R}}{\varepsilon \operatorname{Pe}\left(Q_{1, \text { in }}+Q_{\mathrm{f}, \text { in }}\right)},
$$

we have a non-zero solute concentration throughout the bioreactor. At $\mathrm{O}(\varepsilon)$ we found that, in steady-state, $\bar{c}_{1}$ is constant in time and space. We therefore note from equations (66)-(69) that, given the solution for $c_{0}, g$ is also a constant and hence the first-order outer solute concentrations $c_{i_{1}}(i=1, \mathrm{~m}, \mathrm{w}, \mathrm{f})$ are independent of both $x$ and $t$. We thus investigate how the $y$-dependence of these solutions varies for different values of the inlet fluxes $Q_{1, \text { in }}$ and $Q_{\text {f,in }}$, the cell-scaffold porosity $\phi_{\mathrm{w}}$ and the cell layer width $h_{3}$.

In Figure 4.1, we plot the first-order outer solute concentrations, $c_{i_{1}}(i=1, \mathrm{~m}, \mathrm{w}, \mathrm{f})$, across the entire bioreactor $0<y<H$. The boundaries between each of the four sections are represented by horizontal dotted lines. Given that the constant $\bar{c}_{1}$ (and so $g$ ) could depend on any of our constant parameters (including those being varied), we focus on the qualitative differences which arise as a result of varying the above parameters, noting that the exact value of $\bar{c}_{1}$ (and so $g$ ) only affects the translation of each $c_{i_{1}}$ curve in $y$-space, and not its shape. In order to compare the relative changes, firstly the whole plot is normalised so that the curve corresponding to the middle parameter value (the dashed line in each plot) lies between 0 and 1 . This middle line uses the same parameter values for each of Figure 4.1(a)-(d), so that a direct comparison can be made between these plots. In addition, the other two curves are then translated so that all three curves take the same value at a particular fixed point. The choice of fixed point depends on the parameter being varied, and is chosen in order to make the differences between the relative shape of the curves as clear as possible. We note that this transformation results in 'negative' concentration values being plotted for some parameter choices, but that these values should be used simply to compare the magnitude in variation that arises as a result of these choices.

In each of the plots in Figure 4.1 we observe that, as expected, the concentration is greatest in the lumen and upper fluid layer in each case, with a minimum in the cell layer. The point $y_{\mathrm{c}, \min }$ at which this minimum occurs can be found by setting $\partial c_{\mathrm{w}_{1}} / \partial y=0$ for $c_{0}$ given by equation (115), which yields

$$
y_{\mathrm{c}, \min }=1+h_{2}+\frac{h_{3} Q_{1, \text { in }}}{Q_{1, \text { in }}+Q_{\mathrm{f}, \text { in }}} .
$$

Thus if $Q_{1, \text { in }}=Q_{\mathrm{f}, \text { in }}$, the minimum occurs at $1+h_{2}+h_{3} / 2$, i.e. in the middle of the cell layer. In the two limiting cases of no flow either in the lumen, $Q_{1, \text { in }}=0$, or in the upper fluid layer, 


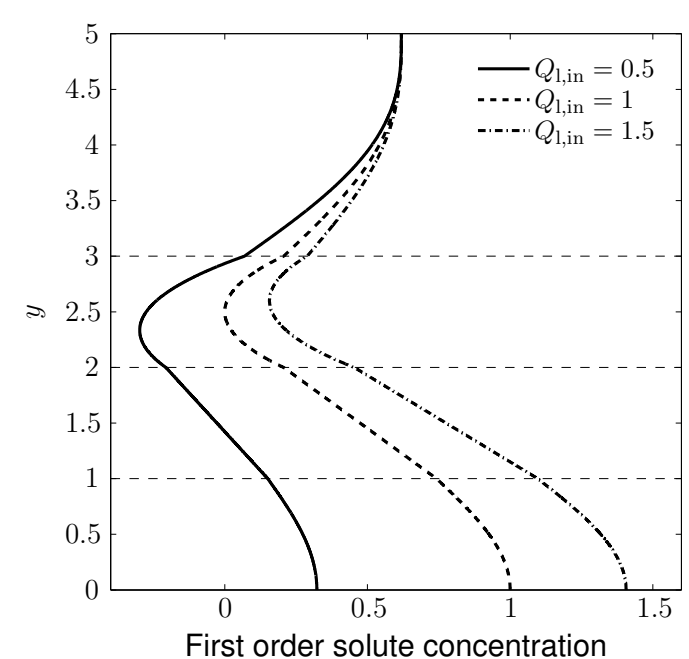

(a)

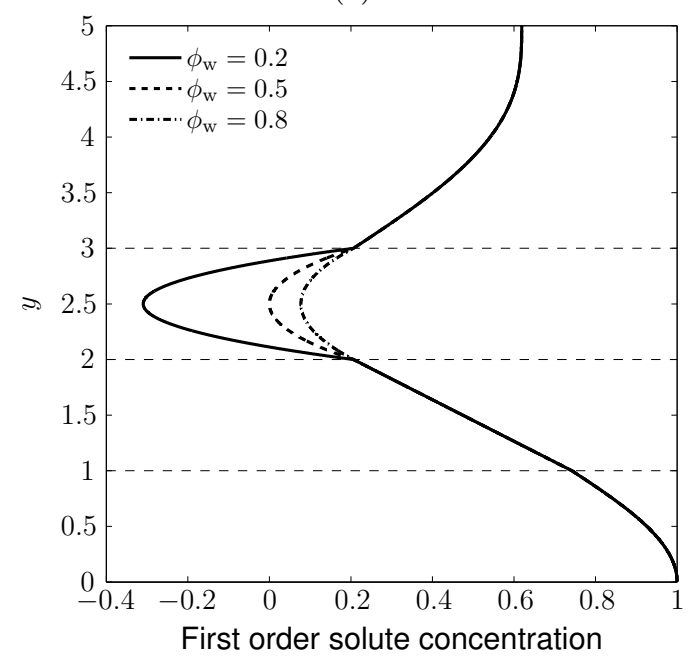

(c)

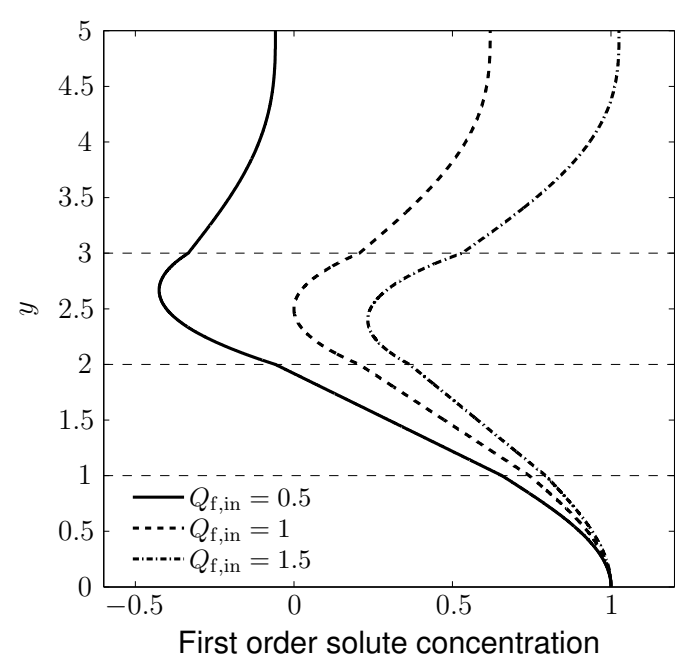

(b)

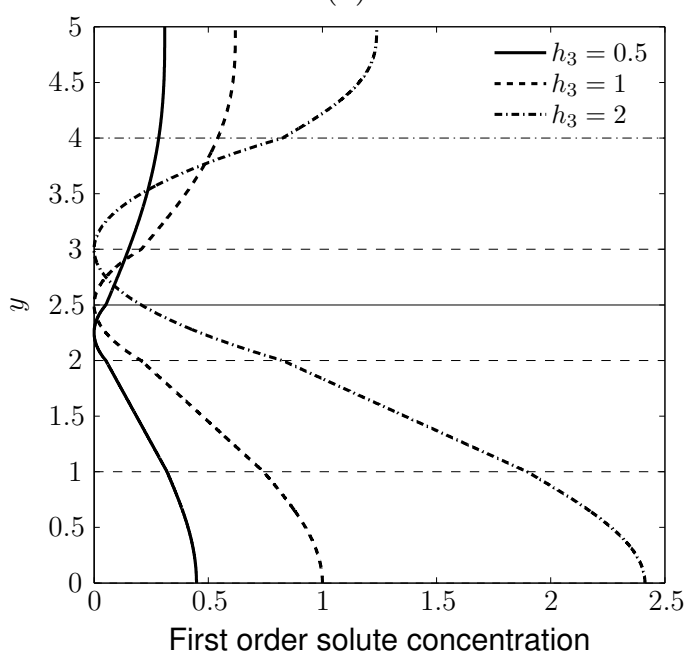

(d)

Figure 4. Plots of the first-order solute concentration in each section for (a) varying lumen inlet flux $Q_{1, \text { in }}$, fixed point $y=5$ (b) varying upper fluid inlet flux $Q_{\mathrm{f}, \text { in }}$, fixed point $y=0$ (c) varying cell layer porosity $\phi_{\mathrm{w}}$, fixed point $y=0$ and (d) varying cell layer thickness $h_{3}$, fixed point minimum of $c_{1}$. In addition to translating to obtain a fixed point common to all 3 lines, plots have been normalised so that the dashed line representing the middle parameter value (which is the same for each plot) always lies between 0 and 1 . Thin dashed lines denote the boundaries between the four sections; in (d) the cell layer/upper fluid interfaces are denoted using the same line style as the corresponding concentration. Fixed parameter values are as in Table II.

$Q_{\mathrm{f}, \text { in }}=0$, the minimum occurs on the membrane/cell layer interface and cell layer/upper fluid interface, respectively.

Figure 4.1(a) shows the trend resulting from varying $Q_{1, \text { in }}$, where the fixed point is the concentration on the top of the bioreactor (at $y=5$ ). As $Q_{1 \text {,in }}$ increases, we see that the concentration in the lumen increases relative to that in the upper fluid layer as the solute is being advected more strongly in the lumen. As would be expected, we then see the lumen concentration decrease relative to that in the upper fluid layer if $Q_{1, \text { in }}$ is decreased. In both of the cases $Q_{1, \text { in }}>Q_{\mathrm{f} \text {,in }}$ and $Q_{1 \text {,in }}<Q_{\mathrm{f} \text {,in }}$, the minimum concentration moves away from the centre of the cell layer, as expected from (117), towards the upper fluid and lumen, respectively. Figure 4.1(b) (where the fixed point is the concentration on the lumen centreline, $y=0$ ) shows that corresponding trends are observed if $Q_{\mathrm{f} \text {,in }}$ is varied, with the upper fluid concentration either increasing or decreasing relative to that in the lumen. We note that the concentration in the 
upper fluid layer is less than that in the lumen when the flow rates are equal. Although the cell layer is in the 'middle' of our two-dimensional domain, the porous membrane acts as a barrier between it and the lumen, so that the effect of the cell uptake on the lumen concentration is less than on the upper fluid concentration. From (66) and (69) we can find the required flux ratio in order for the $\mathrm{O}(\varepsilon)$ concentrations on the lumen centreline and bioreactor top to be equal:

$$
\frac{Q_{1, \text { in }}}{Q_{\mathrm{f}, \text { in }}}=\frac{\frac{h_{3}}{\phi_{\mathrm{w}}}+h_{4}}{2\left(\frac{5}{8}+\frac{h_{2}}{\phi_{\mathrm{m}}}+\frac{h_{3}}{2 \phi_{\mathrm{w}}}\right)} .
$$

Figure 4.1(c) shows the trends arising from varying the cell layer porosity $\phi_{\mathrm{w}}$, again with fixed point the lumen centreline $y=0$. Here, the only qualitative change in concentration occurs in the cell layer, where the concentration decreases relative to elsewhere as $\phi_{\mathrm{w}}$ decreases. This is due to the fact that decreasing $\phi_{\mathrm{w}}$ is equivalent to increasing the cell population density, resulting in more solute being taken up.

Finally, varying the thickness of the cell layer is investigated in Figure 4.1(d), where the fixed point is the minimum concentration value. For lower values of $h_{3}$ there is less overall variation in concentration throughout the bioreactor. This is due to the fact that a thinner cell layer implies less solute is being taken up by the cells. In addition, in agreement with our findings in equation (117), we observe that the minimum concentration is always in the middle of the cell layer.

\subsection{Averaged formulation}

Next we consider features of the unsteady system derived in $\S 3.8$, beginning with the effective diffusion coefficient $D_{\text {eff }}$ and its sensitivity to certain experimentally controlled parameters. We then solve the unsteady system in $\S 4.2 .2$ in order to investigate variations in the mean solute uptake and solute exposure time. The explicit expressions for the concentrations in the case of constant inlet concentration from $\S 4.1$ were used to verify our numerical solutions in $\S 4.2 .2$ (results not shown).

\subsubsection{Effective diffusion coefficient}

The form of $D_{\text {eff }}$ as given in (109) is complex algebraically and its dependence on the main varying parameters $\phi_{\mathrm{m}}, \phi_{\mathrm{w}}, Q_{\mathrm{l} \text {,in }}, Q_{\mathrm{f}, \text { in }}$ and $h_{3}$ is not transparent. In Figure 5 , we have plotted the effective diffusion coefficient for a range of each of these parameters, taking into account the range of validity and any physical constraints in each case. We also plot $D_{\text {eff }}$ for a range of values of the Péclet number Pe to demonstrate the amount of variation obtained for $1 \ll P e \ll \varepsilon^{-2}$. Figure 5 (a) shows that $D_{\text {eff }}$ monotonically decreases with increasing membrane porosity for $0<\phi_{\mathrm{m}} \leq 1$. When the cell layer porosity $\phi_{\mathrm{w}}$ is increased, however, we see that after an initial decrease $D_{\text {eff }}$ reaches a minimum and then increases again as $\phi_{\mathrm{w}}$ approaches 1 (see Figure $5(\mathrm{~b})$ ). Figures 5(c),(d) show the dependence of $D_{\text {eff }}$ on the lumen and upper fluid layer inlet fluxes. For $Q_{1, \text { in }}$ or $Q_{\mathrm{f} \text {,in }}$ sufficiently large ( $\gtrsim 0.63,1.23$ respectively for the parameter values used here), $D_{\text {eff }}$ increases with increasing flux as would be expected from the classical Taylor result. However, below these minimum values, there is a slight increase in $D_{\text {eff }}$ as the flux decreases towards 0 . This is a result of the interaction between the two fluxes, $D_{\text {eff }}$ having terms that depend on $Q_{\mathrm{l} \text {,in }} Q_{\mathrm{f} \text {,in }}$ as well as their respective squares. We note that if we set $Q_{\mathrm{l} \text {,in }}=Q_{\mathrm{f} \text {,in }}$ then $D_{\text {eff }}$ increases monotonically with inlet flux (results not shown). In Figure 5(e) we can see that there is a gradual decrease in $D_{\text {eff }}$ as the cell layer width $h_{3}$ increases to around 1 . For values greater than this, however, the effective diffusion coefficient increases more rapidly with $h_{3}$. This is an interesting observation, but it is not straightforward to determine from the form for $D_{\text {eff }}$ given that increasing $h_{3}$ results in a corresponding decrease of the upper fluid layer $h_{4}$. Finally, Figure $5(\mathrm{f})$ shows the expected combination of linear and inverse dependence of $D_{\text {eff }}$ on Pe. This is 
included for completeness and to show the magnitude of the variation in $D_{\text {eff }}$ which can be obtained by varying Pe.

\subsubsection{Pulse of solute}

We now investigate the behaviour of the unsteady system (107) by considering what happens to a pulse of solute introduced upstream. In order to make analysis of the results more transparent, we will simplify the boundary condition at $x=0^{+}$by taking $\bar{c}_{\mathrm{u}}(t) \equiv 0$ and setting

$$
\bar{c}(t)=\bar{h} c_{\mathrm{in}}(t)=\frac{10}{\sqrt{\pi}} \mathrm{e}^{-100 t^{2}} .
$$

At leading order, this could be interpreted as setting the concentration into the upstream ECS port to be zero, and sending a pulse of solute into the lumen inlet such that (see equation (59))

$$
c_{1, \text { in }}(t)=\frac{\left(Q_{1, \text { in }}+Q_{\mathrm{f}, \text { in }}\right)}{\bar{h} Q_{1, \text { in }}} \frac{10}{\sqrt{\pi}} \mathrm{e}^{-100 t^{2}} .
$$

We close the system with boundary condition (111) as discussed previously. The system (107), (111), (119), (120) was solved in MATLAB using the method of lines and the inbuilt solver ode15s. Given that the order of accuracy of this system is $\mathrm{O}\left(\varepsilon^{2}\right)$, the reaction rate $\mathcal{R}$ was set to be zero for $\bar{c}<\varepsilon^{2}$. Figure 6 shows a typical solution, demonstrating the evolution of the pulse of solute throughout the bioreactor at fixed times. The effect of advection and diffusion on the solute concentration can clearly be seen.

Next, we wish to use this averaged system to investigate how the mean solute uptake and solute exposure time varies with certain parameter values. To do this, we take the 'solute exposure time' $T$ to be the time at which the concentration is less than $\varepsilon^{2}$ throughout the bioreactor, i.e. the time it takes for the pulse of solute to leave the bioreactor, up to the order of accuracy of the system. We then compute the uptake $J(x, t)$ for $0<x<1,0<t<T$ where

$$
J(x, t)= \begin{cases}\frac{h_{3} \mathcal{R}}{\varepsilon \mathrm{Pe}} & \text { if } \quad \bar{c}>\varepsilon^{2} \\ 0 & \text { otherwise. }\end{cases}
$$

We define the mean uptake $\mu$ to be

$$
\mu=\int_{0}^{1} \bar{J}(x) \mathrm{d} x,
$$

where

$$
\bar{J}(x)=\int_{0}^{T} J(x, t) \mathrm{d} t,
$$

and we use the MATLAB function trapz to approximate the integrals using our numerical solution for $\bar{c}$. Figure 7 shows the dependence of $\mu$ and $T$ on the parameter values $\phi_{\mathrm{m}}, \phi_{\mathrm{w}}, Q_{1, \mathrm{in}}$, $Q_{\mathrm{f}, \text { in }}, h_{3}$ and Pe. With the exception of the dependence of $\mu$ on $h_{3}$ and Pe, all graphs demonstrate non-monotonic behaviour, which is likely to arise from the fact that the flow rates, porosities and cell layer width all contribute to both the advection speed $\left(Q_{1, \text { in }}+Q_{\mathrm{f}, \text { in }}\right) / \bar{h}$ and diffusion coefficient $D_{\text {eff }}$. We would expect an increased advection speed to reduce solute exposure time in the bioreactor, and thereby mean uptake, as the cells have less time in which to take up nutrient. On the contrary, we would expect an increased diffusion coefficient to increase the mean uptake, as it results in a more 'spread out' pulse of solute, and thus the cells are exposed to a significant concentration for longer. We also note that the graphs of $\mu$ and $T$ show identical trends for $\phi_{\mathrm{m}}, \phi_{\mathrm{w}}, Q_{\mathrm{l}, \mathrm{in}}$ and $Q_{\mathrm{f}, \text { in }}$, indicating the fact that a longer solute exposure time correlates with a greater mean uptake as would be expected. The same is not true for the dependence on $h_{3}$ 


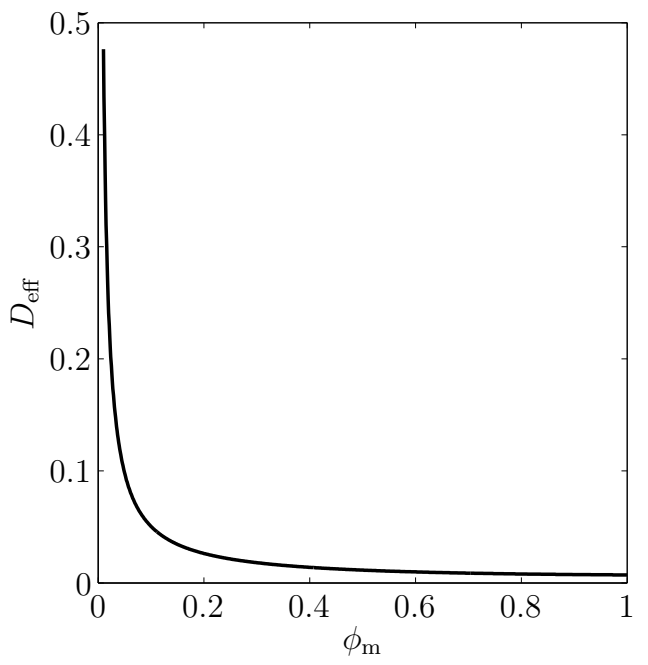

(a)

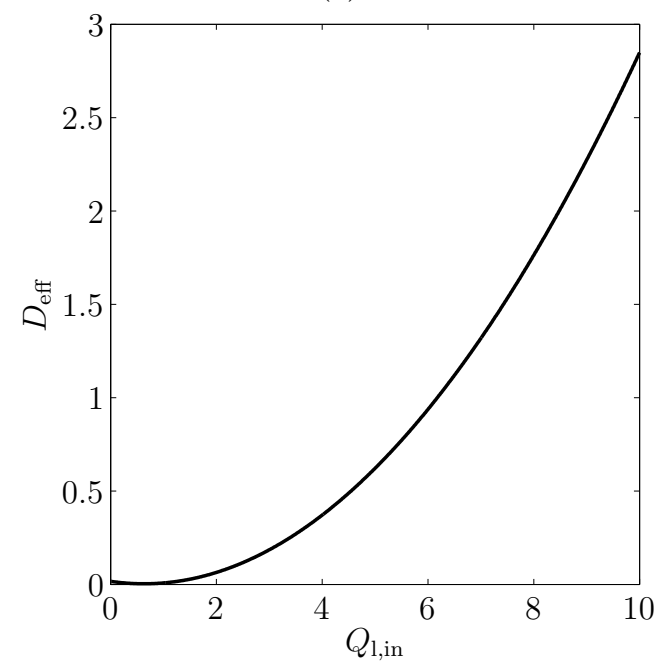

(c)

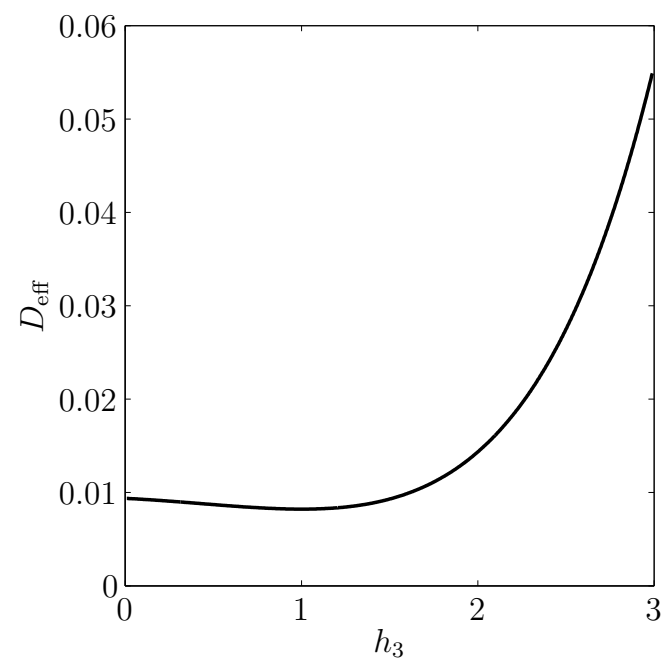

(e)

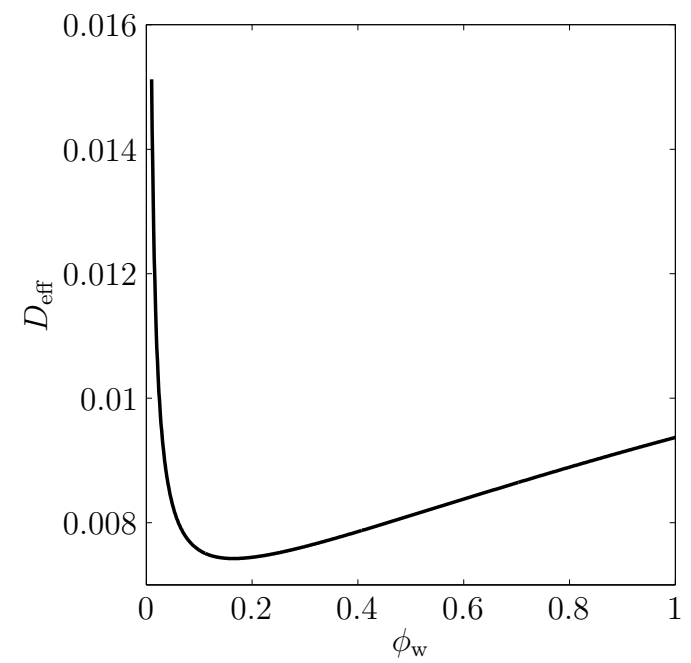

(b)

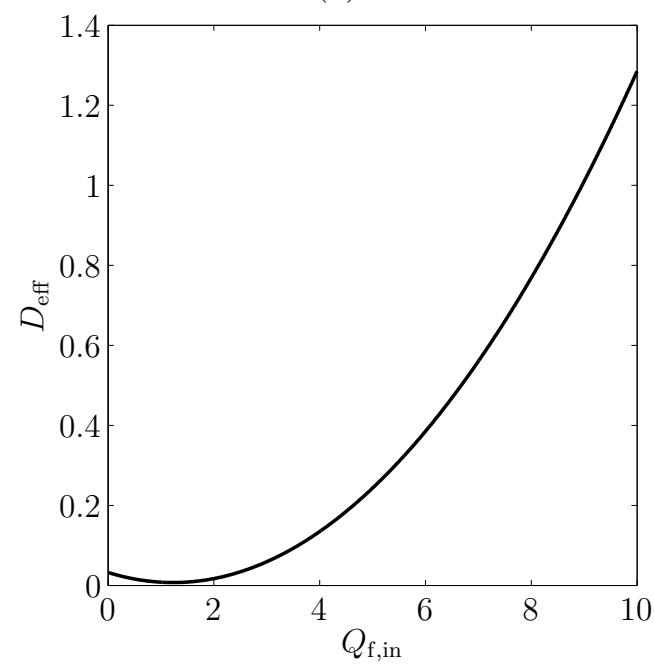

(d)

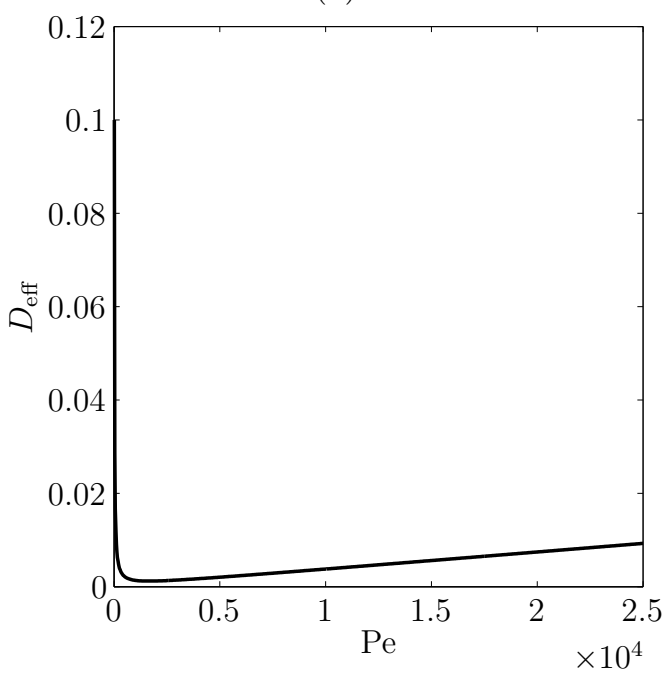

(f)

Figure 5. Plots of $D_{\text {eff }}$ versus (a) the membrane porosity $\phi_{\mathrm{m}}$, (b) the cell layer porosity $\phi_{\mathrm{w}}$, (c) the lumen inlet flux $Q_{1, \text { in }},(\mathrm{d})$ the upper fluid layer inlet flux $Q_{\mathrm{f}, \text { in }}$, (e) the cell layer width $h_{3}$ and (f) the Péclet number Pe. Fixed parameter values are as in Table II. 


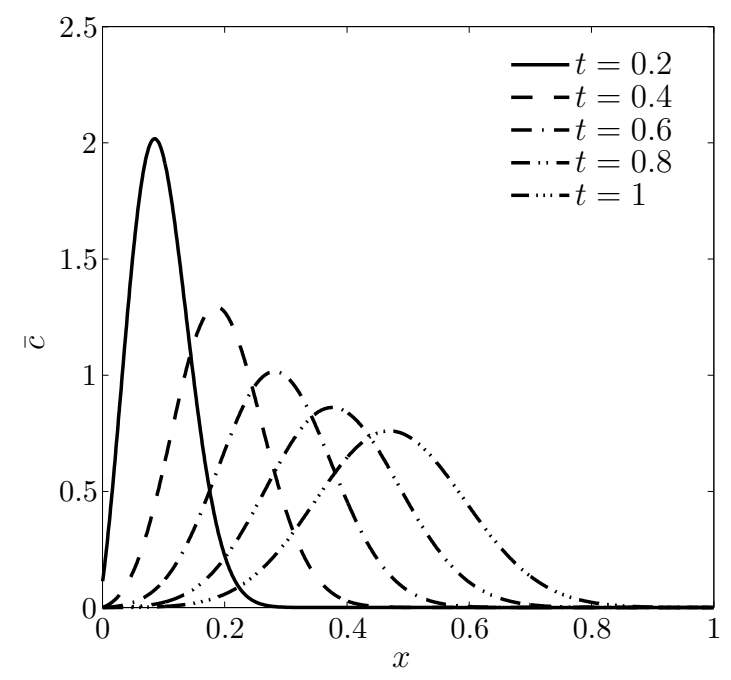

Figure 6. Plot of $\bar{c}$ versus $x$ at fixed times between 0 and 1 , with initial condition given by equation (119). Fixed parameter values are as in Table II.

and Pe; this is due to the fact that these parameter values also affect the rate of solute uptake and so their effect on the system is more complicated. Given a specific experimental setup, this analysis could be used to determine operating parameters which guarantee a high degree of targeted nutrient delivery, which is ideal for generating a viable cell population. It should be noted that the results here are only qualitative in nature, and in order to predict the absolute variation in $\mu$ and $T$, values for the fixed parameters particular to an exact experimental setup would need to be used as inputs for the model, along with generalisation of the two-dimensional geometry presented here to a three-dimensional domain.

\section{Discussion}

In summary, we have developed a two-dimensional model for a simplified HFMB in which the ECS consists of a rigid, porous cell layer below a free-flowing upper layer of fluid. Our aim was to determine the dependence of the solute concentration field on the underlying parameters of the system: the flow rate, solute uptake rate, supplied fluid fluxes, membrane porosity, cell layer porosity and width. By focussing on a timescale characterised by advection in the lumen, cell motility, death and proliferation, all of which occur on longer timescales, can be neglected and the cell layer is assumed to have constant porosity and permeability. A solute, for instance a nutrient such as oxygen, is supplied at both the lumen and upper fluid inlets and is taken up by the cells at a constant rate per unit volume of mixture. The governing equations and boundary conditions have been non-dimensionalised, exploiting the small aspect ratio $\varepsilon$ of the lumen. The flow regime in which the Péclet number $\mathrm{Pe}=\mathrm{O}(1 / \varepsilon)$ is considered, so that advection dominates diffusion in the lumen and upper fluid layer. The system of equations for the fluid flow has been solved analytically at leading- and first-order in this small parameter $\varepsilon$, and these solutions have been used to obtain equations to be solved for the solute concentration at leading- and firstorder. We have identified the appropriate up- and down-stream boundary conditions through analysis of the corresponding inner problems which would need to be solved numerically in order to obtain the exact solution in the inner regions near the up- and down-stream ECS ports. In addition, we have formulated our problem in the context of Taylor dispersion and derived the effective diffusion coefficient governing the dispersion of the averaged solute concentration throughout the bioreactor. The resulting equations have also been shown to simplify to those of 

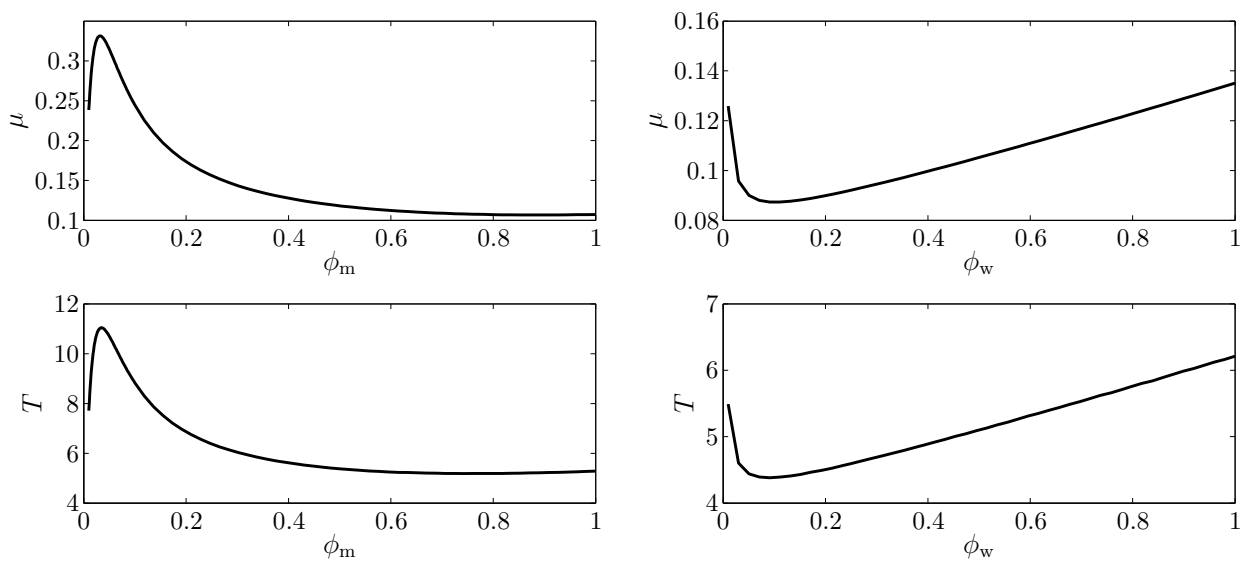

(a)

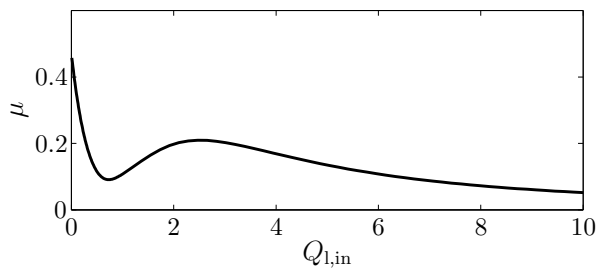

(b)
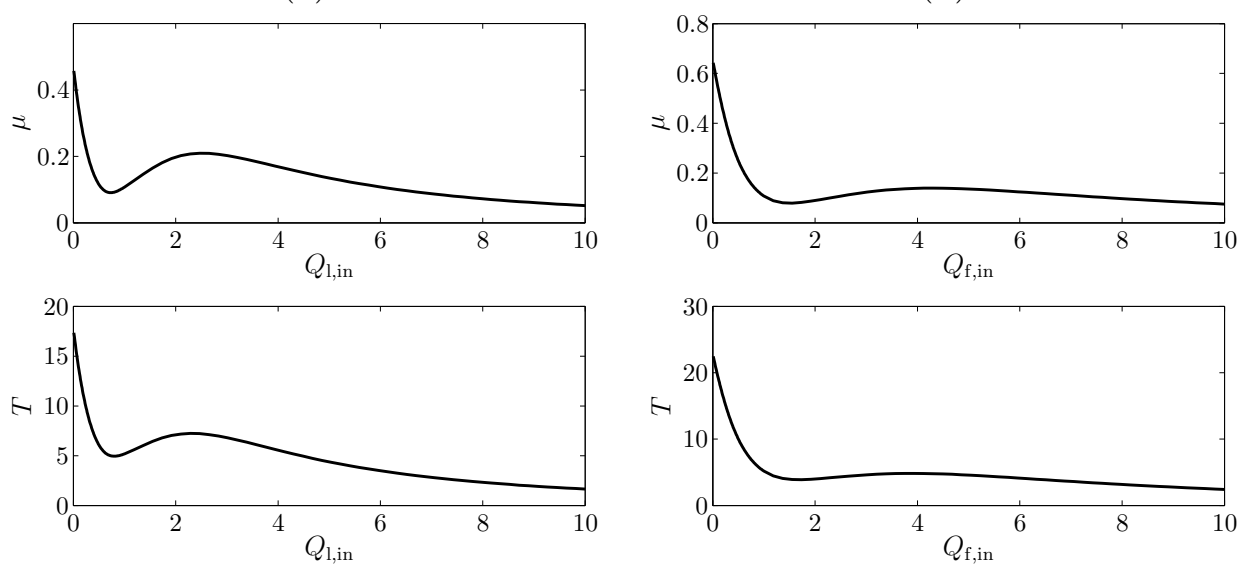

(c)

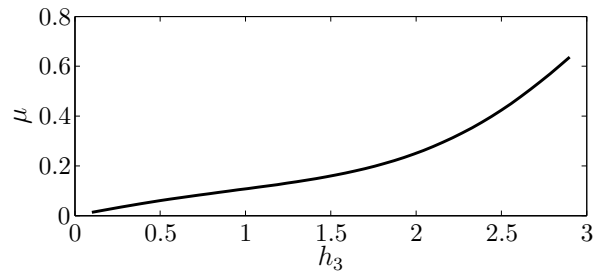

(d)
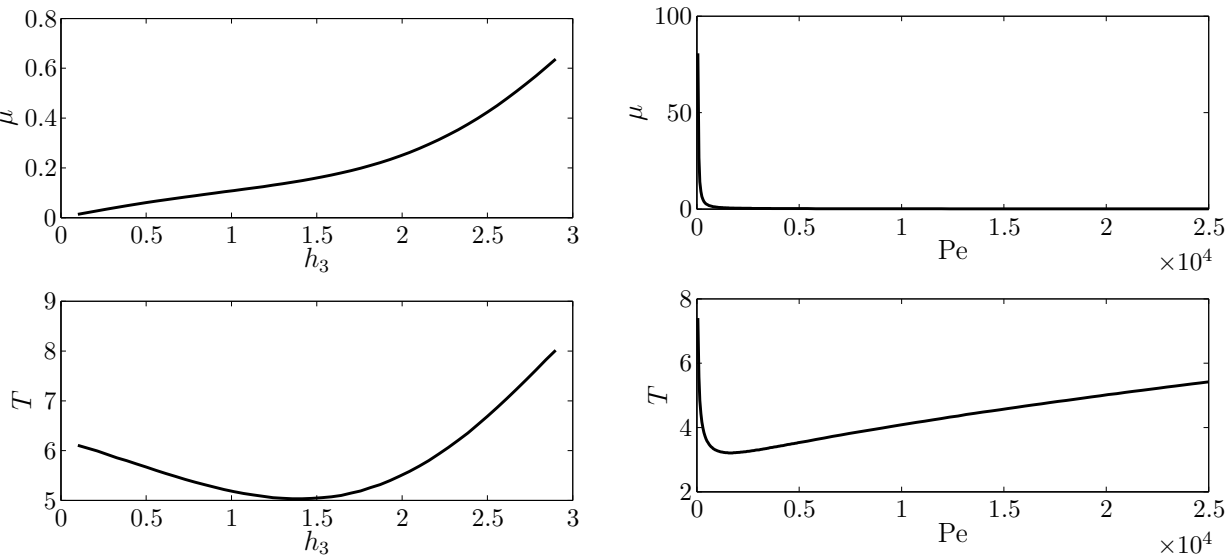

(e)

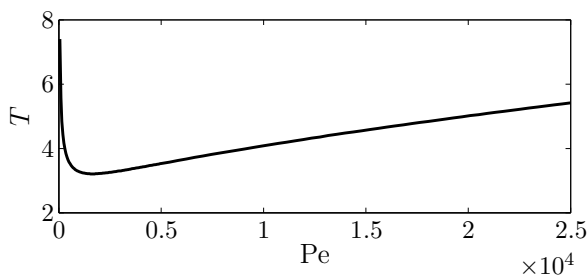

(f)

Figure 7. Plots of the mean solute uptake $\mu$ (top) and solute exposure time $T$ (bottom) versus (a) the membrane porosity $\phi_{\mathrm{m}}$, (b) the cell layer porosity $\phi_{\mathrm{w}}$, (c) the lumen inlet flux $Q_{1, \text { in }}$, (d) the upper fluid layer inlet flux $Q_{\mathrm{f}, \text { in }}$, (e) the cell layer width $h_{3}$ and (f) the Péclet number Pe. Fixed parameter values are as in Table II. 
classical Taylor dispersion in a two-dimensional pipe in the case of an impermeable membrane and cell layer.

We considered the results of the outer asymptotics problem, where it is possible to analytically solve for the outer solute concentration at leading- and first-order in all four sections. From this we determined a lower bound on the inlet concentration $c_{\text {in }}$ which ensures non-zero concentration throughout the bioreactor; the $y$-value at which the minimum concentration is obtained in terms of the cell layer width and inlet fluxes; and the ratio required between the lumen and upper fluid inlet fluxes in order to obtain equal concentrations at the top and bottom of our simplified bioreactor domain, all with an accuracy of $\mathrm{O}\left(\varepsilon^{2}\right)$. Furthermore, we plotted the qualitative trends in the first-order solute concentrations which arise when a number of key dimensionless parameters are varied. We note that to obtain more quantitative results it would be necessary to numerically solve the full inner problem at this order, something which we do not pursue here.

We also investigated the dependence of the effective diffusion coefficient $D_{\text {eff }}$ on these key dimensionless parameters. This revealed complex non-monotonic behaviours in all cases except the dependence on $\phi_{\mathrm{m}}$. These results are due to the interaction of the many different processes involved in the model, as $D_{\text {eff }}$ contains effects from all four sections making up the bioreactor. Finally, we solved the unsteady, combined-order equation in order to investigate the behaviour of a pulse of solute delivered at the inlet. Analysis of the dependence of the mean solute uptake and solute exposure time on key parameter values showed how optimal operating parameters could be determined for a specific experimental setup.

Returning to the question posed in $\S 1$, we have made some progress in determining whether or not the cells receive sufficient nutrient in this setup. Equation (116) gives a minimum inlet concentration required in order to achieve a non-zero nutrient concentration throughout the bioreactor. If we take the nutrient to be oxygen (as in [5]) and wish to check that the concentration is above the minimum required for cell viability (which is equivalent to a dimensionless value of 0.36 , see $[5,8]$ for relevant parameter values), we obtain a more restrictive constraint on the inlet concentration:

$$
c_{\text {in }}>\frac{h_{3} \mathcal{R}}{\varepsilon \operatorname{Pe}\left(Q_{\mathrm{l}, \text { in }}+Q_{\mathrm{f}, \text { in }}\right)}+0.36 .
$$

Both equations (116) and (124) depend on parameters which can be determined from the specific cell and experimental conditions in a particular setup. However, they are both only accurate up to leading order, and may not hold in situations where the $\mathrm{O}(\varepsilon)$ corrections become significant. To find a more accurate constraint in these situations, it would be necessary to solve the upstream inner problem numerically to determine $\bar{c}_{\mathrm{u}}$, as discussed previously. Although we do not undertake this numerical analysis here, we have found an equation for the $y$-value at which the minimum concentration will be attained, again dependent on experimentally obtainable parameters (see (117)), and also qualitative trends to suggest how severe the depletion in the cell layer will be compared with the other sections (see Figure 4.1).

The system considered here is clearly simplified, but nonetheless enables a great deal of information to be obtained analytically regarding the fluid flow and solute concentration in a setup where the Péclet number is large (to be precise, of $\mathrm{O}(1 / \varepsilon)$ ). If we compare the nutrient concentration distributions obtained to those for the nutrient-driven proliferation case study in [5], we see that in both cases we have a leading-order concentration which is independent of $y$. However, here we can find an analytical expression for this concentration, making the dependence on key parameters more transparent. The distribution obtained numerically in the multiphase system in [5] is approximately linear for flow rates at the higher end of the range considered, and hence qualitatively matches the analytical linear distribution found here. In addition, here we can obtain a great deal of information about the next-order correction to the solute distribution which was not possible in the multiphase context; this is important as in this setup (where the flow rates are of $\mathrm{O}(1 / \varepsilon)$ greater than in [5]) this correction term could 
become significant for certain parameter values. Finally, we note that a similar analysis to that presented here can be performed in the three-dimensional case (details of which are omitted), revealing that the flow in the outer region is axisymmetric, at least at leading- and first-order in $\varepsilon$.

\section{Appendix}

\section{A. Inner regions}

In $\S 2$ we introduced a two-dimensional model of a HFMB which included the regions near the up- and down-stream ECS ports. We then discussed the existence of inner regions near these ports in $\S 3$, and focussed on finding the solution valid in the outer region. In $\S \mathrm{A} .1$, we analyse the up- and down-stream inner regions and determine the matching conditions needed to close the outer problem. Furthermore, in Appendix B we consider the boundary layer required in order to close the averaged formulation system from $\S 3.8$.

\section{A.1. Governing EQuations}

We return to the dimensionless equations (19)-(22) and rescale in order to move into the lefthand inner region in which $x=\mathrm{O}(\varepsilon)$. We choose this scaling as it promotes the $x$-derivatives that were neglected in the outer region due to the lubrication scaling, and since we would expect both $x$ - and $y$-derivatives to contribute to the leading-order behaviour in the inner region. Hence we set $x=\varepsilon X$ and $v_{i}=V_{i} / \varepsilon$ (for $i=1, \mathrm{~m}, \mathrm{w}, \mathrm{f}$ ). For clarity we also set , $p_{i}(x, y, t)=P_{i}(X, y, t), c_{i}(x, y, t)=C_{i}(X, y, t), u_{i}(x, y, t)=U_{i}(X, y, t) \quad(i=1, \mathrm{~m}, \mathrm{w}, \mathrm{f})$ and $\nabla_{\mathrm{X}}=\left(\frac{\partial}{\partial X}, \frac{\partial}{\partial y}\right), \nabla_{\mathrm{X}}^{2}=\frac{\partial^{2}}{\partial X^{2}}+\frac{\partial^{2}}{\partial y^{2}}$. We again use subscripts on dependent variables to indicate their domain of applicability from Figure 2. The flow equations are now

$$
\begin{gathered}
\frac{\partial U_{i}}{\partial X}+\frac{\partial V_{i}}{\partial y}=0, \quad-\frac{\partial P_{i}}{\partial X}+\varepsilon \nabla_{\mathrm{X}}^{2} U_{i}=0, \quad-\frac{\partial P_{i}}{\partial y}+\varepsilon \nabla_{\mathrm{X}}^{2} V_{i}=0, \quad i=1, \mathrm{f}, \\
U_{\mathrm{m}}=-\varepsilon \kappa_{\mathrm{m}} \frac{\partial P_{\mathrm{m}}}{\partial X}, \quad V_{\mathrm{m}}=-\varepsilon \kappa_{\mathrm{m}} \frac{\partial P_{\mathrm{m}}}{\partial y}, \quad \nabla_{\mathrm{X}}^{2} P_{\mathrm{m}}=0, \\
U_{\mathrm{w}}=-\varepsilon \kappa_{\mathrm{w}} \frac{\partial P_{\mathrm{w}}}{\partial X}, \quad V_{\mathrm{w}}=-\varepsilon \kappa_{\mathrm{w}} \frac{\partial P_{\mathrm{w}}}{\partial y}, \quad \nabla_{\mathrm{X}}^{2} P_{\mathrm{w}}=0,
\end{gathered}
$$

while the boundary conditions become

$$
\begin{gathered}
\frac{\partial U_{\mathrm{l}}}{\partial y}=0, \quad V_{\mathrm{l}}=0 \quad \text { on } \quad y=0, \\
U_{\mathrm{l}}=0, \quad V_{\mathrm{l}}=-\varepsilon^{2} \kappa_{\mathrm{m}} \phi_{\mathrm{m}} \frac{\partial P_{\mathrm{m}}}{\partial y}, \\
P_{\mathrm{l}}+\frac{2 \varepsilon}{3}\left(\frac{\partial U_{\mathrm{l}}}{\partial X}-2 \frac{\partial V_{\mathrm{l}}}{\partial y}\right)=P_{\mathrm{m}} \quad \text { on } \quad y=1, \\
\phi_{\mathrm{m}} \kappa_{\mathrm{m}} \frac{\partial P_{\mathrm{m}}}{\partial y}=\phi_{\mathrm{w}} \kappa_{\mathrm{w}} \frac{\partial P_{\mathrm{w}}}{\partial y}, \quad P_{\mathrm{m}}=P_{\mathrm{w}} \quad \text { on } \quad y=1+h_{2}, \\
U_{\mathrm{f}}=0, \quad-\varepsilon^{2} \phi_{\mathrm{w}} \kappa_{\mathrm{w}} \frac{\partial P_{\mathrm{w}}}{\partial y}=V_{\mathrm{f}}, \\
P_{\mathrm{w}}=P_{\mathrm{f}}+\frac{2 \varepsilon}{3}\left(\frac{\partial U_{\mathrm{f}}}{\partial X}-2 \frac{\partial V_{\mathrm{f}}}{\partial y}\right) \quad \text { on } \quad y=H-h_{4},
\end{gathered}
$$




$$
U_{\mathrm{f}}=V_{\mathrm{f}}=0 \quad \text { on } \quad y=H,
$$

and, as in $\S 2.2$, we assume that there is a prescribed two-dimensional flux $Q_{1, \text { in }}$ at the lumen inlet, and $Q_{\mathrm{f}, \text { in }}$ at each of the upstream ECS ports. We note that the equations in (125) for the lumen and upper fluid layer are only valid at $\mathrm{O}(1)$ here, since the inertial terms would appear at $\mathrm{O}(\varepsilon)$ (see discussion in $\S 2.1$ ). However, this does not affect this analysis since only the leading-order inner velocities are required, as will be seen below.

Equations $(125 b, c)$ tell us that the leading-order pressure in the lumen and upper fluid layer is independent of both $X$ and $y$. Hence, without needing to solve for the leading- and firstorder velocities in the lumen and upper fluid layer, we can further see from equations (126b), $(127 b)$ that $V_{\mathrm{m}}, V_{\mathrm{w}}=\mathrm{O}\left(\varepsilon^{2}\right)$, and so there is no flux across either the lumen/membrane or cell layer/upper fluid interfaces at $\mathrm{O}(1)$ or $\mathrm{O}(\varepsilon)$.

We first determine the fluid flux conditions by considering how fluid transfers between layers. From inspection of boundary conditions $(129 b)$ and $(131 b)$, we can see that there is no flux of fluid across either the lumen/membrane or cell layer/upper fluid layer interfaces at $\mathrm{O}(1)$ or $\mathrm{O}(\varepsilon)$. Thus the flux out of the inner region in the lumen and upper fluid layer must equal the flux into the outer region up to $\mathrm{O}(\varepsilon)$ in these sections, and hence the correct inlet conditions to impose on the outer flow solution at leading- and first-order in $\S 3.5$ are given by (37), (38), (47) and (51).

The inner problem for the solute concentration is given by

$$
\begin{aligned}
\varepsilon \operatorname{Pe}\left(\varepsilon \frac{\partial C_{\mathrm{l}}}{\partial t}+\nabla_{\mathrm{X}} \cdot\left(C_{\mathrm{l}} \mathbf{U}_{\mathrm{l}}\right)\right) & =\nabla_{\mathrm{X}}^{2} C_{\mathrm{l}}, \\
\varepsilon^{2} \operatorname{Pe}\left(\frac{\partial C_{\mathrm{m}}}{\partial t}+\nabla_{\mathrm{X}} \cdot\left(C_{\mathrm{m}} \mathbf{U}_{\mathrm{m}}\right)\right) & =\nabla_{\mathrm{X}}^{2} C_{\mathrm{m}}, \\
\varepsilon^{2} \operatorname{Pe}\left(\frac{\partial C_{\mathrm{w}}}{\partial t}+\nabla_{\mathrm{X}} \cdot\left(C_{\mathrm{w}} \mathbf{U}_{\mathrm{w}}\right)\right) & =\nabla_{\mathrm{X}}^{2} C_{\mathrm{w}}-\frac{\varepsilon \mathcal{R}}{\phi_{\mathrm{w}}}, \\
\varepsilon \operatorname{Pe}\left(\varepsilon \frac{\partial C_{\mathrm{f}}}{\partial t}+\nabla_{\mathrm{X}} \cdot\left(C_{\mathrm{f}} \mathbf{U}_{\mathrm{f}}\right)\right) & =\nabla_{\mathrm{X}}^{2} C_{\mathrm{f}},
\end{aligned}
$$

for which the boundary conditions are

$$
\begin{array}{rlrlrl}
\frac{\partial C_{\mathrm{l}}}{\partial y} & =0 & & \text { on } y & =0, \\
C_{\mathrm{l}}=C_{\mathrm{m}}, & \frac{\partial C_{\mathrm{l}}}{\partial y} & =\phi_{\mathrm{m}} \frac{\partial C_{\mathrm{m}}}{\partial y} & & \text { on } y=1, \\
C_{\mathrm{m}}=C_{\mathrm{w}}, & \phi_{\mathrm{m}} \frac{\partial C_{\mathrm{m}}}{\partial y} & =\phi_{\mathrm{w}} \frac{\partial C_{\mathrm{w}}}{\partial y} & & \text { on } y=1+h_{2}, \\
C_{\mathrm{w}}=C_{\mathrm{f}}, & \phi_{\mathrm{w}} \frac{\partial C_{\mathrm{w}}}{\partial y} & =\frac{\partial C_{\mathrm{f}}}{\partial y} & & \text { on } y=1+h_{2}+h_{3}, \\
\frac{\partial C_{\mathrm{f}}}{\partial y} & =0 & & \text { on } y=H,
\end{array}
$$

together with the inlet conditions

$$
C_{\mathrm{l}}=c_{1, \text { in }} \quad \text { at } A, \quad C_{\mathrm{f}}=c_{\mathrm{f}, \text { in }} \quad \text { at } B .
$$

As in the outer region, we expand all variables in powers of $\varepsilon$, so that at leading order we obtain (dropping the subscript 0)

$$
\begin{aligned}
& \varepsilon \mathrm{Pe} \nabla_{\mathrm{X}} \cdot\left(C_{\mathrm{l}} \mathbf{U}_{\mathrm{l}}\right)=\nabla_{\mathrm{X}}^{2} C_{\mathrm{l}}, \quad \nabla_{\mathrm{X}}^{2} C_{\mathrm{m}}=0, \\
& \nabla_{\mathrm{X}}^{2} C_{\mathrm{w}}=0, \quad \varepsilon \mathrm{Pe} \nabla_{\mathrm{X}} \cdot\left(C_{\mathrm{f}} \mathbf{U}_{\mathrm{f}}\right)=\nabla_{\mathrm{X}}^{2} C_{\mathrm{f}} .
\end{aligned}
$$


We note that for $c_{1, \text { in }} \neq c_{\mathrm{f} \text {,in }}$ this full inner problem must be solved in order to determine $C_{i}(i=\mathrm{l}, \mathrm{m}, \mathrm{w}, \mathrm{f})$. However, as seen in $\S 3.6$, the leading-order inner concentration must be independent of $y$ as we leave the inner region and therefore can be determined by appealing to global conservation of mass without knowing the solution of this leading-order inner problem. In particular, a far-field analysis of the system (143) subject to (142) consistent with global conservation of mass implies that, as $X \rightarrow \infty$,

$$
C_{i_{0}} \sim c_{\mathrm{in}}=\frac{Q_{1, \text { in }} c_{1, \text { in }}+Q_{\mathrm{f}, \text { in }} c_{\mathrm{f}, \mathrm{in}}}{Q_{1, \mathrm{in}}+Q_{\mathrm{f}, \mathrm{in}}}, \quad i=\mathrm{l}, \mathrm{m}, \mathrm{w}, \mathrm{f}
$$

and hence this gives the correct inlet condition (59) for the $\mathrm{O}(1)$ solute in the outer region.

We now move on to the $\mathrm{O}(\varepsilon)$ problem for the concentration, and note that we do not state the equations for the fluid velocities at this order as we only require the incompressibility condition on $\mathbf{U}_{\mathrm{l}_{1}}$ and $\mathbf{U}_{\mathrm{f}_{1}}$ for our reductions. The $\mathrm{O}(\varepsilon)$ governing equations for the solute concentration are (reintroducing the subscript 0,1 for clarity)

$$
\begin{gathered}
\varepsilon \operatorname{Pe} \nabla_{\mathrm{X}} \cdot\left(C_{\mathrm{l}_{0}} \mathbf{U}_{\mathrm{l}_{1}}+C_{\mathrm{l}_{1}} \mathbf{U}_{\mathrm{l}_{0}}\right)=\nabla_{\mathrm{X}}^{2} C_{\mathrm{l}_{1}}, \quad \nabla_{\mathrm{X}}^{2} C_{\mathrm{m}_{1}}=0, \\
\nabla_{\mathrm{X}}^{2} C_{\mathrm{w}_{1}}=\frac{\mathcal{R}}{\phi_{\mathrm{w}}}, \quad \varepsilon \operatorname{Pe} \nabla_{\mathrm{X}} \cdot\left(C_{\mathrm{f}_{0}} \mathbf{U}_{\mathrm{f}_{1}}+C_{\mathrm{f}_{1}} \mathbf{U}_{\mathrm{f}_{0}}\right)=\nabla_{\mathrm{X}}^{2} C_{\mathrm{f}_{1}} .
\end{gathered}
$$

In general, (145) will need to be solved numerically in order to determine the correct matching condition for the outer solution, something which we do not pursue here. We note, however, that solution of this system would be further complicated by the appearance of $\mathbf{U}_{l_{1}}$ and $\mathbf{U}_{\mathrm{f}_{1}}$, since inertia is not negligible at $\mathrm{O}(\varepsilon)$ in the inner region. However, progress can be made by again considering the far-field limit as $X \rightarrow \infty$.

We begin by making the assumption that the leading-order fluid flow in both the lumen and upper fluid layer is of Poiseuille form as it leaves the inner region. That is, as $X \rightarrow \infty$,

$$
\mathbf{U}_{\mathrm{l}_{0}} \sim\left(\frac{3}{2} Q_{1, \text { in }}\left(1-y^{2}\right), 0\right), \quad \mathbf{U}_{\mathrm{f}_{0}} \sim\left(\frac{6}{h_{4}^{3}} Q_{\mathrm{f}, \text { in }}(y-H)\left(H-h_{4}-y\right), 0\right) .
$$

Substituting these expressions into the far-field limits of the $\mathrm{O}(1)$ lumen and upper fluid layer equations for the solute concentration in (143), we find that the $\mathrm{O}(1)$ far-field concentration is constant in space, and so the terms involving $\mathbf{U}_{\mathrm{l}_{1}}, \mathbf{U}_{\mathrm{f}_{1}}$ disappear as they satisfy the continuity equation. Substituting in the far field forms for $\mathbf{U}_{\mathrm{l}_{0}}$ and $\mathbf{U}_{\mathrm{f}_{0}}$, analysis of (145) subject to the $\mathrm{O}(\varepsilon)$ boundary conditions from (137)-(141) then implies that

$$
\begin{aligned}
C_{\mathrm{l}_{1}} & \sim c_{\infty}(t)-\alpha X+f_{\mathrm{l}}(y), \\
C_{\mathrm{m}_{1}} & \sim c_{\infty}(t)-\alpha X+f_{\mathrm{m}}(y), \\
C_{\mathrm{w}_{1}} & \sim c_{\infty}(T)-\alpha X+f_{\mathrm{w}}(y), \\
C_{\mathrm{f}_{1}} & \sim c_{\infty}(t)-\alpha X+f_{\mathrm{f}}(y),
\end{aligned}
$$


where

$$
\begin{aligned}
& f_{1}=-\frac{\varepsilon \operatorname{Pe} Q_{1, \text { in }} \alpha}{8}\left(6 y^{2}-y^{4}\right)+\frac{\varepsilon \mathrm{Pe}}{2} C_{0}^{\prime}(t) y^{2}+c_{\infty}(t), \\
& f_{\mathrm{m}}=-\varepsilon \operatorname{Pe} Q_{1, \mathrm{in}} \alpha\left(\frac{y-1}{\phi_{\mathrm{m}}}+\frac{5}{8}\right)+\varepsilon \operatorname{Pe} C_{0}^{\prime}(t)\left[\frac{y^{2}}{2}-\left(1-\frac{1}{\phi_{\mathrm{m}}}\right)(y-1)\right]+c_{\infty}(t), \\
& f_{\mathrm{w}}=-\varepsilon \operatorname{Pe} Q_{1, i_{n}} \alpha\left(\frac{y-\left(1+h_{2}\right)}{\phi_{\mathrm{w}}}+\frac{h_{2}}{\phi_{\mathrm{m}}}+\frac{5}{8}\right)+\frac{\mathcal{R}}{2 \phi_{\mathrm{w}}}\left(1+h_{2}-y\right)^{2} \\
& +\varepsilon \operatorname{Pe} C_{0}^{\prime}(t)\left\{\frac{y^{2}}{2}-\left(1+h_{2}\right) y+\left(1+h_{2}\right)^{2}+\frac{\phi_{\mathrm{m}}}{\phi_{\mathrm{w}}}\left(h_{2}+\frac{1}{\phi_{\mathrm{m}}}\right)\left[y-\left(1+h_{2}\right)\right]\right. \\
& \left.-h_{2}\left(1-\frac{1}{\phi_{\mathrm{m}}}\right)\right\}+c_{\infty}(t) \\
& f_{\mathrm{f}}=-\frac{\varepsilon \operatorname{Pe} Q_{\mathrm{f}, \text { in }} \alpha}{h_{4}^{3}} B_{\mathrm{f}}(y)-\varepsilon \operatorname{Pe} Q_{1, \text { in }} \alpha\left(\frac{h_{2}}{\phi_{\mathrm{m}}}+\frac{h_{3}}{\phi_{\mathrm{w}}}+\frac{5}{8}\right) \\
& +\varepsilon \operatorname{Pe} C_{0}^{\prime}(t)\left[\frac{y^{2}}{2}-H y+\left(1+h_{2}\right)^{2}+\left(H-h_{4}\right)\left(h_{3}+h_{4}\right)\right. \\
& \left.+\frac{h_{3} \phi_{\mathrm{m}}}{\phi_{\mathrm{w}}}\left(h_{2}+\frac{1}{\phi_{\mathrm{m}}}\right)-h_{2}\left(1-\frac{1}{\phi_{\mathrm{m}}}\right)\right]+\frac{h_{3}^{2} \mathcal{R}}{2 \phi_{\mathrm{w}}}+c_{\infty}(t), \\
& B_{\mathrm{f}}(y):=-\frac{y^{4}}{2}+\left(2 H-h_{4}\right) y^{3}-3 H\left(H-h_{4}\right) y^{2}-H^{2}\left(3 h_{4}-2 H\right) y \\
& -\frac{H^{4}}{2}+h_{4} H^{3}-\frac{h_{4}^{4}}{2} \\
& \alpha(t)=\frac{h_{3} \mathcal{R}+\varepsilon \operatorname{Pe} \bar{h} C_{0}^{\prime}(t)}{\varepsilon \operatorname{Pe}\left(Q_{\mathrm{l}, \text { in }}+Q_{\mathrm{f}, \text { in }}\right)},
\end{aligned}
$$

and $c_{\infty}(t)$ is a degree of freedom which, through matching with the outer solution, will determine the correct form for $\bar{c}_{\mathrm{u}}$. We note that the leading-order term in each of $C_{i_{1}}(i=\mathrm{l}, \mathrm{m}, \mathrm{w}, \mathrm{f})$ as $X \rightarrow \infty$ corresponds to a linear decay in concentration as a result of uniform uptake across all four sections. This reflects the fact that in the far field of the inner region as $X \rightarrow \infty$, transverse diffusion is sufficiently strong that the dominant contribution to the solute concentration is independent of $y$, as in the outer solution. In order to determine $c_{\infty}(t)$ (and hence $\bar{c}_{\mathrm{u}}(t)$ ) it would be necessary to solve the full inner problem numerically at $O(1)$ and $O(\varepsilon)$. The exact form of $\bar{c}_{\mathrm{u}}$ will not affect the shape of the first-order solute concentrations in the outer region, but will determine their magnitude, and hence whether or not these correction terms make a significant contribution to the leading-order concentration in the outer region.

In the downstream inner region near $x=1$, the same scalings apply and we set $x=1-\varepsilon X$. This gives the same system as the upstream inner region, with sign changes in front of single $X$-derivatives and the downstream boundary conditions

$$
P_{1}=P_{\mathrm{d}} \quad \text { at } C, \quad P_{\mathrm{f}}=0 \quad \text { at } D .
$$

We once again find that the leading-order pressure in the lumen and upper fluid layer is a function of time only, and by the above boundary conditions we can deduce that, at leading order, $P_{1} \equiv P_{\mathrm{d}}$ and $P_{\mathrm{f}} \equiv 0$. By matching with the outer solution as $X \rightarrow \infty$, this gives the correct downstream boundary conditions to apply on the leading-order outer system in $\S 3.5$ as

$$
p_{\mathrm{l}}=P_{\mathrm{d}}, \quad p_{\mathrm{f}}=0 \quad \text { at } x=1^{-} .
$$

This analysis is sufficient for our purposes, however we note that the inner problem here would also need to be solved numerically if the full solution was required; for instance if it was necessary to know the fluid flux and solute concentration leaving the lumen outlet and downstream ECS port in order to match with experimental measurements. 


\section{B. Boundary layer analysis of the combined-order equation}

In this appendix we revisit the combined-order solute concentration equation from $\S 3.8$. We analyse more closely our choice for the additional boundary condition (111),

$$
\frac{\partial \bar{c}}{\partial x}=0 \quad \text { at } \quad x=1^{-},
$$

which is required to close the averaged system (107) and (110):

$$
\begin{gathered}
\frac{\partial \bar{c}}{\partial t}+\frac{Q_{\mathrm{l}, \mathrm{in}}+Q_{\mathrm{f}, \text { in }}}{\bar{h}} \frac{\partial \bar{c}}{\partial x}=D_{\mathrm{eff}} \frac{\partial^{2} \bar{c}}{\partial x^{2}}-\frac{h_{3} \mathcal{R}}{\varepsilon \mathrm{Pe}}, \\
\bar{c}=\bar{h}\left(c_{\mathrm{in}}+\varepsilon c_{\mathrm{u}}\right) \quad \text { at } \quad x=0^{+} .
\end{gathered}
$$

To investigate the error that arises from our choice (159), here we solve the combined-order equation in both the outer region and the boundary layer near $x=1$ up to and including $\mathrm{O}(\varepsilon)$ and compare the resulting composite solution with the globally averaged asymptotic solutions at $\mathrm{O}(1)$ and $\mathrm{O}(\varepsilon)$, namely $\bar{c}_{0}$ and $\bar{c}_{1}$. We assume throughout our analysis that, at the orders considered, the concentration is non-zero throughout the domain (i.e. large enough $t$ and appropriate operating conditions). Otherwise, if the concentration is zero for some $0^{+}<\xi<x<1^{-}$ we note that the boundary condition (159) is trivially satisfied.

We first solve the asymptotic equations and boundary conditions at $O(1)$ and $O(\varepsilon)$ from (100) and write in terms of averaged concentrations. This yields

$$
\begin{aligned}
& \bar{c}_{0}=-\frac{\bar{h} h_{3} \mathcal{R}}{\varepsilon \operatorname{Pe}\left(Q_{1, \text { in }}+Q_{\mathrm{f}, \text { in }}\right)} x+\bar{h} c_{\mathrm{in}}\left(t-\frac{\bar{h}}{Q_{1, \mathrm{in}}+Q_{\mathrm{f}, \mathrm{in}}} x\right), \\
& \bar{c}_{1}=\frac{\bar{h} D_{\mathrm{eff}}}{\varepsilon}\left(\frac{\bar{h}}{Q_{1, \text { in }}+Q_{\mathrm{f}, \mathrm{in}}}\right)^{3} x c_{\mathrm{in}}^{\prime \prime}\left(t-\frac{\bar{h}}{Q_{\mathrm{l}, \mathrm{in}}+Q_{\mathrm{f}, \text { in }}} x\right)+\bar{h} \bar{c}_{\mathrm{u}}\left(t-\frac{\bar{h}}{Q_{1, \text { in }}+Q_{\mathrm{f}, \text { in }}} x\right),
\end{aligned}
$$

where $\bar{c}_{0}=\bar{h} c_{0}$ and ' denotes differentiation with respect to the argument of a function. We define the combined, averaged asymptotic solution up to and including $\mathrm{O}(\varepsilon)$ by $\bar{c}_{\text {as }}:=\bar{c}_{0}+\varepsilon \bar{c}_{1}$, which will be compared to the composite solution to the combined-order equation later.

We now consider the equation for the combined averaged formulation

$$
\frac{\partial \bar{c}}{\partial t}+\frac{Q_{1, \text { in }}+Q_{\mathrm{f}, \text { in }}}{\bar{h}} \frac{\partial \bar{c}}{\partial x}=D_{\mathrm{eff}} \frac{\partial^{2} \bar{c}}{\partial x^{2}}-\frac{h_{3} \mathcal{R}}{\varepsilon \mathrm{Pe}},
$$

and the corresponding conditions

$$
\bar{c}=\bar{h}\left(c_{\mathrm{in}}+\bar{c}_{\mathrm{u}}\right) \quad \text { at } x=0^{+}, \quad \frac{\partial \bar{c}}{\partial x}=0 \quad \text { at } x=1^{-} .
$$

Firstly, we note that if we expand the combined-order concentration $\bar{c}$ in powers of $\varepsilon$ and solve (163) at $\mathrm{O}(1)$ and $\mathrm{O}(\varepsilon)$ subject to the inlet boundary condition at $x=0^{+}$only, this yields 'outer' solutions which are identical to $\bar{c}_{0}$ and $\bar{c}_{1}$ in (161) and (162). For clarity of notation when we form the composite solution later, we will denote this combined-order outer solution by $\bar{c}_{\text {out }}$.

We now wish to find the inner solution in the boundary layer near $x=1$ at $\mathrm{O}(1)$ and $\mathrm{O}(\varepsilon)$. To do so, we rescale

$$
x=1-\varepsilon X \quad(X<0), \quad \bar{c}(x, t)=\bar{C}(X, t),
$$

and (163) then becomes

$$
\varepsilon \frac{\partial \bar{C}}{\partial t}-\frac{Q_{1, \text { in }}+Q_{\mathrm{f}, \text { in }}}{\bar{h}} \frac{\partial \bar{C}}{\partial X}=\frac{D_{\text {eff }}}{\varepsilon} \frac{\partial^{2} \bar{C}}{\partial X^{2}}-\frac{h_{3} \mathcal{R}}{\mathrm{Pe}},
$$


subject to

$$
\frac{\partial \bar{C}}{\partial X}=0 \quad \text { at } \quad X=0
$$

and matching with $\bar{c}$ as $X \rightarrow \infty$. Recalling that $D_{\text {eff }}=\mathrm{O}(\varepsilon)$ and $\mathrm{Pe}=\mathrm{O}(1 / \varepsilon)$, and expanding $\bar{C}$ in powers of $\varepsilon$ with the usual subscript notation, we find at $\mathrm{O}(1)$

$$
\begin{gathered}
-\frac{Q_{1, \text { in }}+Q_{\mathrm{f}, \text { in }}}{\bar{h}} \frac{\partial \bar{C}_{0}}{\partial X}=\frac{D_{\text {eff }}}{\varepsilon} \frac{\partial^{2} \bar{C}_{0}}{\partial X^{2}}, \\
\frac{\partial \bar{C}_{0}}{\partial X}=0 \quad \text { at } \quad X=0 .
\end{gathered}
$$

Solving (168) implies that

$$
\bar{C}_{0}=\alpha(t),
$$

for some function $\alpha(t)$, which can be determined through matching with $\bar{c}_{0}$ as $x \rightarrow 1$ and $X \rightarrow \infty$. Using Van Dyke's rule $(n$ t. o. $)(m$ t. i. $)=(m$ t. i. $)(n$ t. o. $)$ and setting $m=n=1$ gives

$$
\bar{C}_{0}=\alpha(t)=-\frac{\bar{h} h_{3} \mathcal{R}}{\varepsilon \operatorname{Pe}\left(Q_{1, \text { in }}+Q_{\mathrm{f}, \text { in }}\right)}+\bar{h} c_{\text {in }}\left(t-\frac{\bar{h}}{Q_{1, \text { in }}+Q_{\mathrm{f}, \text { in }}}\right) .
$$

At $O(\varepsilon)$ the system to solve is

$$
\begin{gathered}
\frac{\partial \bar{C}_{0}}{\partial t}-\frac{Q_{1, \text { in }}+Q_{\mathrm{f}, \text { in }}}{\bar{h}} \frac{\partial \bar{C}_{1}}{\partial X}=\frac{D_{\text {eff }}}{\varepsilon} \frac{\partial^{2} \bar{C}_{1}}{\partial X^{2}}-\frac{h_{3} \mathcal{R}}{\varepsilon \mathrm{Pe}}, \\
\frac{\partial \bar{C}_{1}}{\partial X}=0 \quad \text { at } \quad X=0,
\end{gathered}
$$

which has solution

$$
\begin{aligned}
\bar{C}_{1}=\frac{\bar{h}}{Q_{1, \text { in }}+Q_{\mathrm{f}, \text { in }}} & {\left[\frac{D_{\text {eff }} \bar{h}}{\varepsilon\left(Q_{1, \text { in }}+Q_{\mathrm{f}, \text { in }}\right)} \exp \left(-\frac{\varepsilon\left(Q_{1, \text { in }}+Q_{\mathrm{f}, \text { in }}\right)}{\bar{h} D_{\text {eff }}} X\right)\right.} \\
+ & \left.X-\frac{D_{\text {eff }} \bar{h}}{\varepsilon\left(Q_{1, \text { in }}+Q_{\mathrm{f}, \text { in }}\right)}\right]\left[\bar{h} c_{\text {in }}^{\prime}\left(t-\frac{\bar{h}}{Q_{1, \text { in }}+Q_{\mathrm{f}, \text { in }}}\right)+\frac{h_{3} \mathcal{R}}{\varepsilon \operatorname{Pe}}\right]+\beta(t),
\end{aligned}
$$

for some function $\beta(t)$. Matching is automatically satisfied for $m=2, n=1$ and $m=1, n=2$, and for $m=n=2$ we find

$$
\begin{aligned}
& \beta(t)=\frac{D_{\text {eff }}}{\varepsilon}\left(\frac{\bar{h}}{Q_{1, \text { in }}+Q_{\mathrm{f}, \text { in }}}\right)^{2}\left[\bar{h} c_{\text {in }}^{\prime}\left(t-\frac{\bar{h}}{Q_{1, \text { in }}+Q_{\mathrm{f}, \text { in }}}\right)+\frac{h_{3} \mathcal{R}}{\varepsilon \mathrm{Pe}}\right. \\
& \left.+\frac{\bar{h}^{2}}{Q_{1, \text { in }}+Q_{\mathrm{f}, \text { in }}} c_{\text {in }}^{\prime \prime}\left(t-\frac{\bar{h}}{Q_{1, \text { in }}+Q_{\mathrm{f}, \text { in }}}\right)\right]+\bar{h} \bar{c}_{\mathrm{u}}\left(t-\frac{\bar{h}}{Q_{1, \text { in }}+Q_{\mathrm{f}, \mathrm{in}}}\right) .
\end{aligned}
$$

From (170) and (172) we can thus obtain the combined-order inner solution, $\bar{C}_{\text {in }}:=\bar{C}_{0}+\varepsilon \bar{C}_{1}$ and then also construct the composite solution to the combined-order equation,

$$
\bar{c}_{\text {comp }}:=\bar{c}_{\text {out }}+\bar{C}_{\text {in }}-\bar{c}_{\text {over }},
$$

where $\bar{c}_{\text {over }}$ is the 'overlap' between the outer and inner solutions, found from the (2 t. i.) $(2$ t. o.) terms. We can now compare the composite solution $\bar{c}_{\text {comp }}$ to the combined-order equation (163) to the combined, averaged asymptotic solution, $\bar{c}_{\text {as }}$. Given the analysis presented here, we expect the two solutions to match up to $\mathrm{O}\left(\varepsilon^{2}\right)$, except in the boundary layer of width $\mathrm{O}(\varepsilon)$ near $x=1$ within which we expect an error of $\mathrm{O}(\varepsilon)$. Plots comparing the two solutions confirm that this is the case (results not shown), and hence (159) is an appropriate choice of boundary condition for the combined-order system in $\S 3.8$. 


\section{Acknowledgements}

N.C.P. acknowledges funding by the EPSRC in the form of a studentship at the Systems Biology Doctoral Training Centre, University of Oxford.

\section{References}

1. Stock UA, Vacanti JP (2001) Tissue engineering: Current state and prospects. Annu Rev Med 52(1):443-451

2. Pörtner R, Nagel-Heyer S, Goepfert C, Adamietz P, Meenen NM (2005) Bioreactor design for tissue engineering. J Biosci Bioeng 100(3):235-245

3. Martin I, Wendt D, Heberer M (2004) The role of bioreactors in tissue engineering. Trends Biotechnol 22(2):80-86

4. Martin Y, Vermette P (2005) Bioreactors for tissue mass culture: Design, characterization, and recent advances. Biomaterials 26(35):7481-7503

5. Pearson NC, Shipley RJ, Waters SL, Oliver JM (2013) Multiphase modelling of the influence of fluid flow and chemical concentration on tissue growth in a hollow fibre membrane bioreactor. Math Med Biol doi: 10.1093/imammb/dqt015

6. Personal communication with Dr. Marianne Ellis, Centre for Regenerative Medicine, University of Bath

7. Shipley RJ, Davidson AJ, Chan K, Chaudhuri JB, Waters SL, Ellis MJ (2011) A strategy to determine operating parameters in tissue engineering hollow fiber bioreactors. Biotechnol Bioeng 108(6):1450-1461

8. Shipley RJ, Waters SL (2012) Fluid and mass transport modelling to drive the design of cell-packed hollow fibre bioreactors for tissue engineering applications. Math Med Biol 29:329-359

9. Aris R (1956) On the dispersion of a solute in a fluid flowing through a tube. P Roy Soc Lond A Mat 235(1200):67-77

10. Taylor G (1953) Dispersion of soluble matter in solvent flowing slowly through a tube. P Roy Soc Lond A Mat 219(1137):186-203

11. Taylor G (1954) Conditions under which dispersion of a solute in a stream of solvent can be used to measure molecular diffusion. P Roy Soc Lond A Mat 225(1163):473-477

12. Griffiths IM, Howell PD, Shipley RJ (2013) Control and optimization of solute transport in a thin porous tube. Phys Fluids (1994-present) 25(3):033101-1-18

13. Kumar JP, Umavathi JC, Chamkha AJ, Basawaraj A (2012) Solute dispersion between two parallel plates containing porous and fluid layers. J Porous Media 15(11):1031-1047

14. Pal D, Veerabhadraiah R, Shivakumar PN, Rudraiah N (1984) Longitudinal dispersion of tracer particles in a channel bounded by porous media using slip condition. Internat J Math Math Sci 7(4):755-764

15. Vikhansky A, Wang W (2011) Taylor dispersion in finite-length capillaries. Chem Eng Sci 66(4):642-649

16. Mazumder BS, Mondal KK (2005) On solute transport in oscillatory flow through an annular pipe with a reactive wall and its application to a catheterized artery. Q J Mech Appl Math 58(3):349-365

17. Mondal KK, Mazumder B (2005) On the solute dispersion in a pipe of annular cross-section with absorption boundary. Z Angew Math Mech 85(6):422-430

18. Van den Broeck C, Dekempeneer E (1983) The effect of boundary absorption on longitudinal dispersion in steady laminar flows. Z Angew Math Phys 34(4):489-501

19. Shipley RJ, Waters SL, Ellis MJ (2010) Definition and validation of operating equations for poly(vinyl alcohol)-poly(lactide-co-glycolide) microfiltration membrane-scaffold bioreactors. Biotechnol Bioeng 107(2):382-392

20. Lemon G, King JR, Byrne HM, Jensen OE, Shakesheff KM (2006) Mathematical modelling of engineered tissue growth using a multiphase porous flow mixture theory. J Math Biol 52:571-594

21. Meneghello G, Parker DJ, Ainsworth BJ, Perera SP, Chaudhuri JB, Ellis MJ, De Bank PA (2009) Fabrication and characterization of poly(lactic-co-glycolic acid)/polyvinyl alcohol blended hollow fibre membranes for tissue engineering applications. J Membr Sci 344(1-2):55-61

22. O'Dea RD, Waters SL, Byrne HM (2010) A multiphase model for tissue construct growth in a perfusion bioreactor. Math Med Biol 27(2):95-127

23. Aris R (1959) On the dispersion of a solute by diffusion, convection and exchange between phases. P Roy Soc Lond A Mat 252(1271):538-550 